\title{
The validity of Whitham's approximation for a Klein-Gordon-Boussinesq model
}

\author{
Wolf-Patrick Düll, Kourosh Sanei Kashani, Guido Schneider \\ Institut für Analysis, Dynamik und Modellierung, Universität Stuttgart, \\ Pfaffenwaldring 57, 70569 Stuttgart, Germany
}

October 5, 2018

\begin{abstract}
In this paper we prove the validity of a long wave Whitham approximation for a system consisting of a Boussinesq equation coupled with a Klein-Gordon equation. The proof is based on an infinite series of normal form transformations and an energy estimate. We expect that the concepts of this paper will be a part of a general approximation theory for Whitham's equations which are especially used in the description of slow modulations in time and space of periodic wave trains in general dispersive wave systems.
\end{abstract}

\section{Introduction}

For dispersive wave systems there are various long wave approximations, the most prominent ones are KdV approximation and the Whitham approximation. For the Boussinesq equation

$$
\partial_{t}^{2} u=\partial_{x}^{2} u+\partial_{t}^{2} \partial_{x}^{2} u+\partial_{x}^{2}\left(u^{2}\right)
$$

with $x, t, u(x, t) \in \mathbb{R}$, as a toy model, with the ansatz

$$
u(x, t)=\varepsilon^{2} A\left(\varepsilon(x-t), \varepsilon^{3} t\right),
$$

where $0<\varepsilon \ll 1$ is a small perturbation parameter and $A(X, T) \in \mathbb{R}$, we obtain the KdV equation

$$
\partial_{T} A=\partial_{X}^{3} A / 2+\partial_{X}\left(A^{2}\right) / 2,
$$


and with the ansatz

$$
u(x, t)=A(\varepsilon x, \varepsilon t),
$$

again with $A(X, T) \in \mathbb{R}$, we obtain the Whitham system

$$
\partial_{t}^{2} u=\partial_{x}^{2} u+\partial_{x}^{2}\left(u^{2}\right)
$$

which can be written as a first order system

$$
\partial_{t} u=\partial_{x} v, \quad \partial_{t} v=\partial_{x} u+\partial_{x}\left(u^{2}\right)
$$

of conservation laws. The approximation of solutions of a dispersive system like the Boussinesq equation (1) by solutions to the KdV equation and the Whitham system, respectively, is called KdV approximation and Whitham approximation, respectively.

Both approximations describe the modes which are concentrated at the wave number $k=0$ in Fourier space. See Figure 1 .

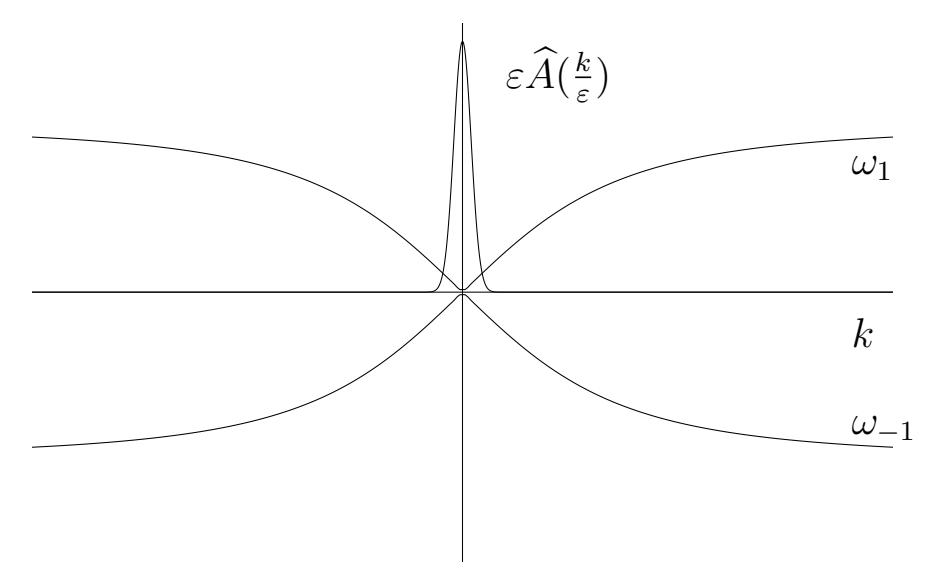

Figure 1: Curves of eigenvalues $\omega_{ \pm 1}$ for the Boussinesq model (1). Since the Fourier transform of $\varepsilon^{2} A(\varepsilon x)$ is given by $\varepsilon \widehat{A}\left(\frac{k}{\varepsilon}\right)$ the modes of the KdV and Whitham approximation are strongly concentrated at the wave number $k=0$.

The error made by these approximations can easily be estimated in both cases by simple energy estimates, cf. [SU16, §20], similar to [GS01] by using the long wave character of the approximation. The fact that the nonlinear terms vanish at the wave number $k=0$, too, allows to construct an energy which allows to control the $\mathcal{O}\left(\varepsilon^{2}\right)$ terms on the $\mathcal{O}\left(1 / \varepsilon^{3}\right)$ time scale, respectively, to control the $\mathcal{O}(1)$ terms on the $\mathcal{O}(1 / \varepsilon)$ time scale. For the justification of the $\mathrm{KdV}$ approximation this approach 
has been used for the water wave problem in Cra85, SW00a, SW02, D12] and for the FPU system in [SW00b].

The system changes dramatically if more than the two curves of eigenvalues, as drawn in Figure 2, are present in the problem. Examples are the water wave problem over a periodic bottom topography, the poly-atomic FPU model and slow modulations in time and space of periodic wave trains. The simplest toy problem with this property is a system consisting of a Boussinesq equation coupled with a Klein-Gordon equation, namely

$$
\begin{aligned}
& \partial_{t}^{2} u=\partial_{x}^{2} u+\partial_{t}^{2} \partial_{x}^{2} u+\partial_{x}^{2}\left(u^{2}+2 u v+v^{2}\right), \\
& \partial_{t}^{2} v=\partial_{x}^{2} v-2 v-\left(u^{2}+2 u v+v^{2}\right),
\end{aligned}
$$

with $x, t, u(x, t), v(x, t) \in \mathbb{R}$. This set of equations is called KGB system in the following. The linearization of the KGB model around the trivial solution $u=v=0$ has plane wave solutions of the form $u(x, t)=e^{i k x+i \omega_{ \pm 1}(k) t}$ and $v(x, t)=e^{i k x+i \omega_{ \pm 2}(k) t}$ with

$$
\omega_{ \pm 1}(k)= \pm \operatorname{sign}(\mathrm{k}) \sqrt{\frac{\mathrm{k}^{2}}{\mathrm{k}^{2}+1}} \quad \text { and } \quad \omega_{ \pm 2}(\mathrm{k})= \pm \sqrt{\mathrm{k}^{2}+2} .
$$

The curves of eigenvalues are plotted in Figure 2.

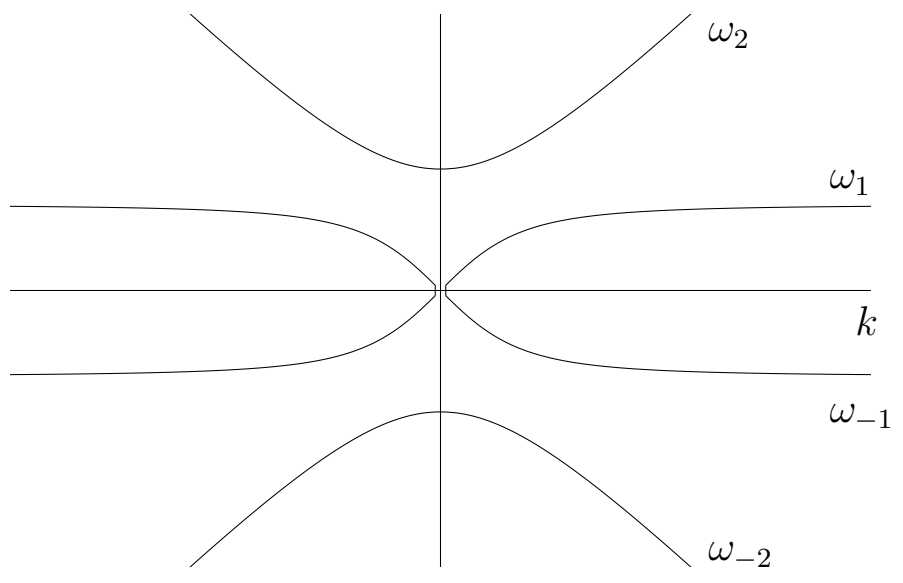

Figure 2: Curves of eigenvalues $\omega_{ \pm 1}$ and $\omega_{ \pm 2}$ of the linearized KGB model

In this situation simple energy estimates in general are no longer sufficient to justify the long wave approximations since now quadratic terms are present in the problem which no longer vanish at the wave number $k=0$. For a KdV approximation with a combination of one normal form transformation and energy estimates this 
problem has been solved. See [CS11] for the KGB system and CCPS12, GMWZ14] for the poly-atomic FPU model.

For the Whitham approximation a new serious difficulty occurs, namely the fact that due to the scaling of the ansatz for the derivation of the Whitham system infinitely many normal form transformations have to be performed instead of only one in the $\mathrm{KdV}$ case. It is the goal of this paper to show that this approach really works and that an approximation theorem for the Whitham approximation of the KGB system can be established.

The ansatz for the derivation of the Whitham system from the KGB system has the form

$$
\psi_{u}^{\text {Whitham }}(x, t)=U(\varepsilon x, \varepsilon t) \text { and } \psi_{v}^{\text {Whitham }}(x, t)=V(\varepsilon x, \varepsilon t) .
$$

Inserting this ansatz into (2) and (3) we find for

$$
\begin{aligned}
& \operatorname{Res}_{u}(u, v)=-\partial_{t}^{2} u+\partial_{x}^{2} u+\partial_{t}^{2} \partial_{x}^{2} u+\partial_{x}^{2}\left(u^{2}+2 u v+v^{2}\right), \\
& \operatorname{Res}_{v}(u, v)=-\partial_{t}^{2} v+\partial_{x}^{2} v-2 v-\left(u^{2}+2 u v+v^{2}\right)
\end{aligned}
$$

that

$$
\begin{aligned}
& \operatorname{Res}_{u}\left(\psi_{u}^{\text {Whitham }}, \psi_{v}^{\text {Whitham }}\right)=\varepsilon^{2}\left(-\partial_{T}^{2} U+\partial_{X}^{2} U+\partial_{X}^{2}\left(U^{2}+2 U V+V^{2}\right)\right)+\varepsilon^{4} \partial_{T}^{2} \partial_{X}^{2} U, \\
& \operatorname{Res}_{v}\left(\psi_{u}^{\text {Whitham }}, \psi_{v}^{\text {Whitham }}\right)=-2 V-\left(U^{2}+2 U V+V^{2}\right)+\varepsilon^{2}\left(-\partial_{T}^{2} V+\partial_{X}^{2} V\right) .
\end{aligned}
$$

Hence equating the coefficients of $\varepsilon^{0}$ in $\operatorname{Res}_{v}$ to zero yields

$$
2 V+U^{2}+2 U V+V^{2}=0,
$$

and so $V=H(U)=-U^{2} / 2+\mathcal{O}\left(U^{3}\right)$ due to the implicit function theorem for $U$ and $V$ of $\mathcal{O}(1)$, but sufficiently small. Equating the coefficients of $\varepsilon^{2}$ in $\operatorname{Res}_{u}$ to zero gives

$$
-\partial_{T}^{2} U+\partial_{X}^{2} U+\partial_{X}^{2}\left(U^{2}+2 U V+V^{2}\right)=0
$$

By substituting $V=H(U)$ into (5) we find

$$
-\partial_{T}^{2} U+\partial_{X}^{2} U+\partial_{X}^{2}\left(U^{2}+2 U H(U)+H(U)^{2}\right)=0 .
$$

Rewriting (6) in conservation law form as

$$
\begin{aligned}
\partial_{T} U & =\partial_{X} W \\
\partial_{T} W & =\partial_{X}\left(U+U^{2}+2 U H(U)+H(U)^{2}\right)
\end{aligned}
$$

yields Whitham's equations.

It is the purpose of this paper to prove the following approximation result. 
Theorem 1.1. There exist $C_{1}>0, \varepsilon_{0}>0$ and $C_{2}>0$ such that the following holds. Let $U \in C\left(\left[0, T_{0}\right], H^{5}(\mathbb{R}, \mathbb{R})\right)$ be a solution of (6) with $\sup _{T \in\left[0, T_{0}\right]}\|U(\cdot, T)\|_{H^{5}} \leq C_{1}$ and let $V=H(U)$. Then for all $\varepsilon \in\left(0, \varepsilon_{0}\right)$ we have solutions $(u, v)$ of (2)-(3) such that

$$
\sup _{t \in\left[0, T_{0} / \varepsilon\right]} \sup _{x \in \mathbb{R}}|(u, v)(x, t)-(U, V)(\varepsilon x, \varepsilon t)| \leq C_{2} \varepsilon^{3 / 2} .
$$

Remark 1.2. The $H^{5}$-control of $U$ is needed to estimate the residual generated by an improved approximation ansatz in $H^{1}$, see Lemma 2.2. More generally, if we have $U \in C\left(\left[0, T_{0}\right], H^{s+4}(\mathbb{R}, \mathbb{R})\right)$ with $\sup _{T \in\left[0, T_{0}\right]}\|U(\cdot, T)\|_{H^{s+4}} \leq C_{1}$ for $s \geq 1$, then our proof of Theorem 1.1 yields the error estimate

$$
\sup _{t \in\left[0, T_{0} / \varepsilon\right]}\|(u, v)(\cdot, t)-(U, V)(\varepsilon \cdot, \varepsilon t)\|_{H^{s}} \leq C_{2} \varepsilon^{3 / 2} .
$$

The question occurs whether to a given initial condition the associated solution of the KGB system can be approximated by a Whitham approximation. This will be discussed in Section 5 .

In order to explain why infinitely many normal from transformations have to be performed in the proof of Theorem 1.1 we explain the strategy of our proof in more detail. We write system (2)-(3) as a first order evolutionary system of the form

$$
\partial_{t} \mathcal{W}=\Lambda \mathcal{W}+B(\mathcal{W}, \mathcal{W})
$$

where $\Lambda$ is a linear skew symmetric operator and $B$ is a bilinear symmetric mapping. By adding higher order terms to the approximation (4) we construct in Section 2 an approximation $\psi$ which is $\mathcal{O}\left(\varepsilon^{2}\right)$-close to $\left(\psi_{u}^{\text {Whitham }}, \psi_{v}^{\text {Whitham }}\right)$ and satisfies formally

$$
\operatorname{Res}(\psi)=-\partial_{t} \psi+\Lambda \psi+B(\psi, \psi)=\mathcal{O}\left(\varepsilon^{4}\right) .
$$

The error function $R$ defined by $\mathcal{W}(x, t)=\psi(x, t)+\varepsilon^{\beta} R(x, t)$ fulfills

$$
\partial_{t} R=\Lambda R+2 B(\psi, R)+\varepsilon^{\beta} B(R, R)+\varepsilon^{-\beta} \operatorname{Res}(\psi) .
$$

To prove an $\mathcal{O}(1)$-bound for $R$ on an $\mathcal{O}(1 / \varepsilon)$-time scale we have to control the terms on the right hand side on this long time scale. The first term is skew-symmetric and will lead to oscillations without any growth rates. The last term can be $\mathcal{O}(\varepsilon)$ bounded if $\beta \leq 3$. If $\beta$ is chosen larger than 1 the third terms gives a bound smaller than $\mathcal{O}(\varepsilon)$. However, the second term $2 B(\psi, R)$ is only $\mathcal{O}(1)$-bounded and can lead to $\mathcal{O}(1)$ growth rates. In order to show that an $\mathcal{O}\left(e^{t}\right)$ growth does not occur we use 
normal form transformations and energy estimates. Normal form transformations are near identity changes of variables of the form

$$
R_{1}=R+M(\psi, R)
$$

where $M$ is a suitably chosen bilinear mapping. Eliminating the term $B(\psi, R)$ by such a normal form transformation is only possible if the non-resonance condition

$$
\inf _{j, n \in\{ \pm 1, \pm 2\}, k \in \mathbb{R}}\left|\omega_{j}(k)-\omega_{1}(0)-\omega_{n}(k)\right|>0
$$

is satisfied, which is not the case for the KGB model since $\omega_{1}(0)=0$. But since the less restrictive non-resonance condition

$$
\inf _{k \in \mathbb{R}}\left\{\left|\omega_{ \pm 1}(k)-\omega_{1}(0)-\omega_{ \pm 2}(k)\right|,\left|\omega_{ \pm 2}(k)-\omega_{1}(0)-\omega_{ \pm 1}(k)\right|\right\}>0
$$

is true for the KGB model the term $B(\psi, R)$ can be split into a resonant and a non-resonant part, i.e.,

$$
B(\psi, R)=B_{\text {res }}(\psi, R)+B_{\text {non }}(\psi, R)
$$

and the non-resonant part $B_{\text {non }}(\psi, R)$ can be eliminated by a normal form transformation. After the normal form transformation in the equation for the new error function $R_{1}$ new terms of order $\mathcal{O}(1)$ appear. They can be split again into resonant and non-resonant terms. Another normal form transformation is necessary to eliminate these new non-resonant terms, but again terms of $\mathcal{O}(1)$ are created. However, they are cubic w.r.t. $\psi$. This goes ad infinitum and so the convergence of the composition of these infinitely many transformations has to be proven. Since the $n$-th transformation is of order $\mathcal{O}\left(\|\psi\|^{n}\right)$ the convergence finally can be established with the help of the geometric series for $\|\psi\|=\mathcal{O}(1)$, but sufficiently small w.r.t. some $\|\cdot\|$-norm. After all these transformations the equation for the transformed error $R_{\infty}$ takes the form

$$
\partial_{t} R_{\infty}=\Lambda R_{\infty}+F\left(\psi, R_{\infty}\right)+\mathcal{O}(\varepsilon)
$$

where $F$ is a function which is linear w.r.t. $R_{\infty}$ and which contains infinitely many resonant terms. Since all these terms now in contrast to the original $B(\psi, R)$ have a long-wave character w.r.t. $t$, i.e., these terms depend explicitly only on $\varepsilon t$ and not on $t$, a suitably chosen energy $E\left(R_{\infty}\right)$ satisfies

$$
\frac{d}{d t} E\left(R_{\infty}\right)=\mathcal{O}(\varepsilon)
$$


and so an $\mathcal{O}(1)$-bound for the transformed error $R_{\infty}$ and the original error $R$, respectively, can be established on the $\mathcal{O}\left(\varepsilon^{-1}\right)$-time scale by applying Gronwall's inequality. The series of normal form transformations can be found in Section 3 and the energy estimates in Section 4 .

We close this introduction with a number of remarks

Remark 1.3. Proving Theorem 1.1 is a nontrivial task since we have to prove an $\mathcal{O}(1)$-bound for the error on an $\mathcal{O}(1 / \varepsilon)$-time scale. There exists a number of counter examples [Sch95, SSZ15] where a formally derived amplitude equation makes wrong predictions about the dynamics of the original system.

Remark 1.4. Whitham's equations belong to the class of generic and universal amplitude equations containing the KdV equation, the NLS equation, the GinzburgLandau equation, Burgers' equation, and so-called phase diffusion equations. Amplitude equations play an important role in the description of spatially extended conservative or dissipative physical systems, where they can be formally derived with the help of a multiple scaling ansatz. Whitham's equations are especially used to describe slow modulations in time and space of periodic wave trains in dispersive wave systems. There exist a series of approximation results for the Ginzburg-Landau approximation, for instance in CE90, vH91, Sch94a, Sch94b, for the KdV approximation, for instance in [Cra85, SW00a, SW02, D12, and for the NLS approximation, for instance in [Kal87, Sch98, Sch05, BSTU06, DS06, TW12, DSW16]. Approximation results for so-called phase diffusion equations, Burgers equation or conservation laws describing modulations of periodic waves in dissipative systems can be found in [MS04b, MS04a, DSSS09]. In the conservative case, i.e. for Whitham's equations, the first nonlinear approximation result has been established in [DS09], namely the validity of Whitham's equations for the NLS equation as original system. However, the spectral picture of the problem considered in [DS09] is as drawn in Figure 1 and not as in Figure 2.

Remark 1.5. Whitham derived his equations first in [Whi65a, Whi65b] and they are still a subject of active research, cf. [DHM06, M06. They are an amplitude system for which so far there has not been established a satisfying theory which shows mathematically rigorously that the original system behaves approximately as predicted by the associated amplitude equation.

Remark 1.6. The Boussinesq equation (2) is a model equation for the water wave problem, whereas the solution of the Klein-Gordon equation (3) represents a scalar quantum field. Here we ignore this origin and couple them solely with the goal to obtain a spectral picture as plotted in Figure 2. 
Remark 1.7. More generally, Whitham's equations are universal approximation equations for large classes of nonlinear PDEs of periodic wave type, see for example [DS09]. Very often they are derived from the Lagrangian of the underlying problem leading to a system of conservation laws, similar to (7)-(8). As mentioned above, the resonance structure of the KGB model is the same as in the situation in which one is really interested in, namely the description of slow modulations in time and space of a periodic traveling wave in a dispersive wave system. By linearizing around the periodic wave in a co-moving frame, we obtain an eigenvalue problem which is periodic in the spatial variable. Its solutions are given by Bloch modes $e^{\mathrm{i} l x+\mathrm{i} \omega_{n}(l) t} v_{n}(l, x)$ with $n \in \mathbb{Z} \backslash\{0\}, l \in\left[\frac{-1}{2 L}, \frac{1}{2 L}\right)$, and where $v_{n}$ possesses the same periodicity $L$ w.r.t. $x$ as the periodic wave. The curves $l \mapsto \omega_{n}(l)$ are ordered by $\omega_{n}(l) \leq \omega_{n+1}(l)$ and by $\omega_{n}(l)=-\omega_{-n}(l)$. In general we have $\omega_{ \pm 1}(0)=0$ and $\omega_{ \pm 2}(0) \neq 0$ since in such systems the periodic wave is accompanied by an at least two-dimensional family of periodic waves. Whitham's equations describe the dynamics of the modes associated with the two curves $\omega_{ \pm 1}$ in the limit $l \rightarrow 0$, see Figure 2 .

Remark 1.8. As explained above we think that our analysis is a necessary step for the validity of Whitham's equations in the general situation. However, before applying these ideas a number of additional questions have to be answered, most essential: how to extract the wave numbers in non $S^{1}$-symmetric systems such that these satisfy equations which are suitable for existing functional analytic tools?

Remark 1.9. Recently Whitham's equations have been in the focus of investigations concerning modulations of periodic wave trains in dissipative systems containing conservation laws [JNRZ14]. The problems with quadratic resonances addressed in the present work do not appear in the dissipative situation. We expect that the analysis for a justification result in the sense of Theorem 1.1 in the dissipative situation is very similar to the one given in [DSSS09, Section 6] where a single conservation law has been justified as an amplitude equation.

Remark 1.10. We expect that an underlying Hamiltonian structure may allow to find an energy which allows to perform a justification analysis of the Whitham approximation without the need of infinitely many transformations. However, such an approach strongly depends on the problem. In contrast, the method presented here is rather independent of the special original system in this class of problems and hence more robust.

Notation. Possibly different constants that can be chosen independent of $0<$ $\varepsilon \ll 1$ are denoted by the same symbol $C$. From now on we write $\int$ instead of $\int_{-\infty}^{\infty}$. The space $H_{m}^{s}$ consists of $s$-times weakly differentiable functions for which 
$\|u\|_{H_{m}^{s}}=\left\|u \rho^{m}\right\|_{H^{s}}=\left(\sum_{j=0}^{s} \int\left|\partial_{x}^{j}\left(u \rho^{m}\right)\right|^{2}(x) d x\right)^{1 / 2}$, with $\rho(x)=\sqrt{1+x^{2}}$, is finite, where we do not distinguish between scalar and vector-valued functions or real- and complex-valued functions. We use $H^{s}$ as an abbreviation for $H_{0}^{s}$. Moreover, we use the space $L_{m}^{1}$ with the norm $\|u\|_{L_{m}^{1}}=\left\|u \rho^{m}\right\|_{L^{1}}$. The Fourier transform of a function $u$ is denoted by

$$
(\mathcal{F} u)(k)=\widehat{u}(k)=\frac{1}{2 \pi} \int u(x) e^{-i k x} d x
$$

and is an isomorphism between $H_{m}^{s}$ and $H_{s}^{m}$. The point-wise multiplication $(u v)(x)=$ $u(x) v(x)$ in $x$-space corresponds to the convolution

$$
(\widehat{u} * \widehat{v})(k)=\int \widehat{u}(k-l) \widehat{v}(l) d l
$$

in Fourier space.

Acknowledgments: The paper is partially supported by the Deutsche Forschungsgemeinschaft DFG under the grant Schn520/9. The authors thank Mariana Haragus, James Kennedy and an unknown referee for their useful comments.

\section{The improved approximation and estimates for the residual}

As explained above we need the residual to be small. With the approximation defined in (4) we formally find that $\operatorname{Res}_{u}\left(\psi_{u}^{\text {Whitham }}, \psi_{v}^{\text {Whitham }}\right)=\mathcal{O}\left(\varepsilon^{4}\right)$, but only $\operatorname{Res}_{v}\left(\psi_{u}^{\text {Whitham }}, \psi_{v}^{\text {Whitham }}\right)=\mathcal{O}\left(\varepsilon^{2}\right)$. In order to have $\operatorname{Res}_{v}=\mathcal{O}\left(\varepsilon^{4}\right)$, too, we extend the ansatz (4) to

$$
\psi_{u}(x, t)=U(\varepsilon x, \varepsilon t) \quad \text { and } \quad \psi_{v}(x, t)=V(\varepsilon x, \varepsilon t)+\varepsilon^{2} V_{2}(\varepsilon x, \varepsilon t) .
$$

We find

$$
\begin{aligned}
\operatorname{Res}_{v}\left(\psi_{u}, \psi_{v}\right)= & -V-\left(U^{2}+2 U V+V^{2}\right)+\varepsilon^{2}\left(-\partial_{T}^{2} V+\partial_{X}^{2} V-V_{2}-2 U V_{2}-2 V V_{2}\right) \\
& +\varepsilon^{4}\left(-\partial_{T}^{2} V_{2}+V_{2}^{2}+\partial_{X}^{2} V_{2}\right) .
\end{aligned}
$$

We formally obtain $\operatorname{Res}_{v}\left(\psi_{u}, \psi_{v}\right)=\mathcal{O}\left(\varepsilon^{4}\right)$ by choosing

$$
V_{2}=\frac{\partial_{X}^{2} V-\partial_{T}^{2} V}{1+2 U+2 V}
$$

For $U$ and $V$ sufficiently small, but still of order $\mathcal{O}(1)$, the function $V_{2}$ is well-defined. 
Remark 2.1. In the following we estimate the difference between a true solution of (2)-(3) and the improved approximation defined in (11). The estimate for the difference between a true solution of (2)-(3) and the original approximation defined in (4) then follows by the triangle inequality using

$$
\sup _{t \in\left[0, T_{0} / \varepsilon\right]} \sup _{x \in \mathbb{R}}\left|\left(\psi_{u}, \psi_{v}\right)(x, t)-\left(\psi_{u}^{\text {Whitham }}, \psi_{v}^{\text {Whitham }}\right)(x, t)\right| \leq C \varepsilon^{2} .
$$

The difference between a true solution of (2)-(3) and the improved approximation defines the error functions $R_{u}$ and $R_{v}$ by

$$
\varepsilon^{\beta} R_{u}=u-\psi_{u} \quad \text { and } \quad \varepsilon^{\beta} R_{v}=v-\psi_{v}
$$

with a suitably chosen $\beta$. The error functions satisfy

$$
\begin{aligned}
& \partial_{t}^{2} R_{u}=\partial_{x}^{2} R_{u}+\partial_{t}^{2} \partial_{x}^{2} R_{u}+2 \partial_{x}^{2}\left(\psi_{u} R_{u}+\psi_{v} R_{u}+\psi_{u} R_{v}+\psi_{v} R_{v}\right) \\
& +\varepsilon^{\beta} \partial_{x}^{2}\left(R_{u}^{2}+2 R_{u} R_{v}+R_{v}^{2}\right) \\
& +\varepsilon^{-\beta} \underbrace{\left(-\partial_{t}^{2} \psi_{u}+\partial_{x}^{2} \psi_{u}+\partial_{t}^{2} \partial_{x}^{2} \psi_{u}+\partial_{x}^{2}\left(\psi_{u}^{2}+2 \psi_{u} \psi_{v}+\psi_{v}^{2}\right)\right)}_{=\operatorname{Res}_{u}\left(\psi_{u}, \psi_{v}\right)}, \\
& \partial_{t}^{2} R_{v}=\partial_{x}^{2} R_{v}-R_{v}-2\left(R_{u} \psi_{u}+R_{u} \psi_{v}+R_{v} \psi_{u}+R_{v} \psi_{v}\right) \\
& -\varepsilon^{\beta}\left(R_{u}^{2}+2 R_{u} R_{v}+R_{v}^{2}\right) \\
& +\varepsilon^{-\beta} \underbrace{\left(-\partial_{t}^{2} \psi_{v}+\partial_{x}^{2} \psi_{v}-\psi_{v}-\left(\psi_{u}^{2}+2 \psi_{u} \psi_{v}+\psi_{v}^{2}\right)\right)}_{=\operatorname{Res}_{v}\left(\psi_{u}, \psi_{v}\right)},
\end{aligned}
$$

where the residual terms are formally of order $\mathcal{O}\left(\varepsilon^{4}\right)$. These equations will be solved in some Sobolev spaces. Estimating the residual terms in these Sobolev spaces will lose $\varepsilon^{-1 / 2}$ due to the scaling properties of the $L^{2}$-norm, namely

$$
\left(\int|U(\varepsilon x)|^{2} d x\right)^{1 / 2}=\left(\varepsilon^{-1} \int|U(X)|^{2} d X\right)^{1 / 2}
$$

and so we have the following lemma.

Lemma 2.2. For $s \geq 1$ there exist $C_{1}>0, \varepsilon_{0}>0$ and $C_{2}>0$ such that the following holds. Let $U \in C\left(\left[0, T_{0}\right], H^{s+4}(\mathbb{R}, \mathbb{R})\right)$ be a solution of $(6)$ with $\sup _{T \in\left[0, T_{0}\right]}\|U(\cdot, T)\|_{H^{s+4}}$ $\leq C_{1}$, let $V=H(U)$, and let $V_{2}$ be defined in $(12)$. Then for all $\varepsilon \in\left(0, \varepsilon_{0}\right)$ we have

$$
\sup _{t \in\left[0, T_{0} / \varepsilon\right]}\left(\left\|\operatorname{Res}_{u}\left(\psi_{u}, \psi_{v}\right)\right\|_{H^{s}}+\left\|\operatorname{Res}_{v}\left(\psi_{u}, \psi_{v}\right)\right\|_{H^{s}}\right)<C_{2} \varepsilon^{7 / 2}
$$


Proof. Combining the formal calculations from above with the scaling properties (15) of the $L^{2}$-norm yields the required estimates. In order to avoid losing more powers of $\varepsilon$ in products arising in $\operatorname{Res}_{u, v}$ only one factor is estimated in $H^{s}$. All others are estimated in $C_{b}^{s}$. The assumption $U(\cdot, T) \in H^{s+4}(\mathbb{R}, \mathbb{R})$ is necessary to estimate $\partial_{X}^{2} V_{2} \in H^{s}(\mathbb{R}, \mathbb{R})$ via $V_{2}=\mathcal{O}\left(\partial_{X}^{2} V\right)$ due to 12 .

When writing (13)-(14) as a first order system we additionally need

Lemma 2.3. Under the assumptions of Lemma 2.2 for all $\varepsilon \in\left(0, \varepsilon_{0}\right)$ we have

$$
\sup _{t \in\left[0, T_{0} / \varepsilon\right]}\left(\left\|\omega_{1}^{-1} \operatorname{Res}_{u}\left(\psi_{u}, \psi_{v}\right)\right\|_{H^{s}}+\left\|\omega_{2}^{-1} \operatorname{Res}_{v}\left(\psi_{u}, \psi_{v}\right)\right\|_{H^{s}}\right)<C_{2} \varepsilon^{5 / 2} .
$$

Proof. The terms of the residual have either spatial derivatives in front or are time derivatives which can be expressed via (6) as terms with spatial derivatives in front. Hence, in Fourier space all terms of the residual have at least a factor $k$ and so the application of $\omega_{1}(k)^{-1}$ to these terms is well-defined. However, because of the long-wave character of the ansatz (11) there is a loss of $\mathcal{O}\left(\varepsilon^{-1}\right)$ since one derivative is canceled by the application of $\omega_{1}(k)^{-1}$. Hence, the assertion of the lemma follows from Lemma 2.2.

\section{The series of normal form transformations}

In order to establish the validity of Theorem 1.1 we have to prove an $\mathcal{O}(1)$-bound for $R_{u}$ and $R_{v}$ on an $\mathcal{O}\left(\varepsilon^{-1}\right)$ time scale. Therefore we need to control the terms on the right hand sides of (13) and (14) on this long time scale. As already said, the main part of this paper is devoted to the handling of the linear $\psi$-dependent terms. Therefore, the error equations are rewritten in the form

$$
\begin{aligned}
& \partial_{t}^{2} R_{u}=\partial_{x}^{2} R_{u}+\partial_{t}^{2} \partial_{x}^{2} R_{u}+2 \partial_{x}^{2}\left(\psi_{u} R_{u}+\psi_{v} R_{u}+\psi_{u} R_{v}+\psi_{v} R_{v}\right)+\varepsilon p_{u, 1} \\
& \partial_{t}^{2} R_{v}=\partial_{x}^{2} R_{v}-2 R_{v}-2\left(\psi_{u} R_{u}+2 \psi_{v} R_{u}+\psi_{u} R_{v}+\psi_{v} R_{v}\right)+\varepsilon p_{v, 1}
\end{aligned}
$$

where the terms $p_{u, 1}$ and $p_{v, 1}$ are defined by

$$
\begin{aligned}
& \varepsilon p_{u, 1}=\varepsilon^{\beta} \partial_{x}^{2}\left(R_{u}^{2}+2 R_{u} R_{v}+R_{v}^{2}\right)+\varepsilon^{-\beta} \operatorname{Res}_{u} \\
& \varepsilon p_{v, 1}=-\varepsilon^{\beta}\left(R_{u}^{2}+2 R_{u} R_{v}+R_{v}^{2}\right)+\varepsilon^{-\beta} \operatorname{Res}_{v} .
\end{aligned}
$$

These last terms provide high enough orders w.r.t. $\varepsilon$ such that they cause no difficulties in arriving at the $\mathcal{O}\left(\varepsilon^{-1}\right)$ time scale if we choose $\beta=3 / 2$. Because of Lemma 2.2 we have

$$
\left\|\varepsilon p_{u, 1}\right\|_{H^{s}}+\left\|\varepsilon p_{v, 1}\right\|_{H^{s+2}} \leq C\left(\varepsilon^{3 / 2}\left(\left\|R_{u}\right\|_{H^{s+2}}+\left\|R_{v}\right\|_{H^{s+2}}\right)^{2}+\varepsilon^{2}\right) .
$$


We write (16)-(17) as a first order system, which in Fourier space has the form

$$
\begin{aligned}
\partial_{t} \widehat{R}_{u} & =i \omega_{1} \widehat{W}_{u} \\
\partial_{t} \widehat{W}_{u} & =i \omega_{1} \widehat{R}_{u}+2 i \omega_{1}\left(\widehat{\psi}_{u} * \widehat{R}_{u}+\widehat{\psi}_{v} * \widehat{R}_{u}+\widehat{\psi}_{u} * \widehat{R}_{v}+\widehat{\psi}_{v} * \widehat{R}_{v}\right)+\varepsilon \widehat{p}_{u, 2}, \\
\partial_{t} \widehat{R}_{v} & =i \omega_{2} \widehat{W}_{v} \\
\partial_{t} \widehat{W}_{v} & =i \omega_{2} \widehat{R}_{v}+2 i \omega_{2}^{-1}\left(\widehat{\psi}_{u} * \widehat{R}_{u}+\widehat{\psi}_{v} * \widehat{R}_{u}+\widehat{\psi}_{u} * \widehat{R}_{v}+\widehat{\psi}_{v} * \widehat{R}_{v}\right)+\varepsilon \widehat{p}_{v, 2},
\end{aligned}
$$

with $\widehat{p}_{u, 2}(k, t)=-\varepsilon^{-1} i \omega_{1}^{-1}(k) \frac{1}{k^{2}+1} \widehat{p}_{u, 1}(k, t)$ and $\widehat{p}_{v, 2}(k, t)=-\varepsilon^{-1} i \omega_{2}^{-1}(k) \widehat{p}_{v, 1}(k, t)$, where $\widehat{p}_{u, j}$ and $\widehat{p}_{v, j}$ are the Fourier transform of $p_{u, j}$ and $p_{v, j}$. Since the nonlinear terms in (13) have two spatial derivatives in front, in Fourier space they are $\mathcal{O}\left(k^{2}\right)$, and so the application of $\omega_{1}(k)^{-1}$ is well-defined for all terms containing $\widehat{R}_{u}$ and $\widehat{R}_{v}$. Since the $\varepsilon$-order of $\widehat{R}_{u}$ and $\widehat{R}_{v}$, in contrast to the residual terms, purely comes from the amplitude and not from the long-wave character of the ansatz (11) the application of $\omega_{1}(k)^{-1}$ causes no loss of $\mathcal{O}\left(\varepsilon^{-1}\right)$ for all terms containing $\widehat{R}_{u}$ and $\widehat{R}_{v}$. For the terms coming from the residual we now use Lemma 2.3 instead of Lemma 2.2 and obtain

$$
\left\|\varepsilon \widehat{p}_{u, 2}\right\|_{H_{s}^{0}}+\left\|\varepsilon \widehat{p}_{v, 2}\right\|_{H_{s}^{0}} \leq C\left(\varepsilon^{3 / 2}\left(\left\|\widehat{R}_{u}\right\|_{H_{s}^{0}}+\left\|\widehat{R}_{v}\right\|_{H_{s}^{0}}\right)^{2}+\varepsilon\right) .
$$

We diagonalize (18)-(19) with

$$
\left(\begin{array}{c}
\widehat{R}_{u} \\
\widehat{W}_{u}
\end{array}\right)=\frac{1}{\sqrt{2}}\left(\begin{array}{cc}
1 & 1 \\
1 & -1
\end{array}\right)\left(\begin{array}{c}
\widehat{R}_{1} \\
\widehat{R}_{-1}
\end{array}\right),\left(\begin{array}{c}
\widehat{R}_{v} \\
\widehat{W}_{v}
\end{array}\right)=\frac{1}{\sqrt{2}}\left(\begin{array}{cc}
1 & 1 \\
1 & -1
\end{array}\right)\left(\begin{array}{c}
\widehat{R}_{2} \\
\widehat{R}_{-2}
\end{array}\right)
$$

and find

$$
\begin{aligned}
\partial_{t} \widehat{R}_{1} & =i \omega_{1} \widehat{R}_{1}+i \omega_{1}\left(S_{1}\left(\Psi, \widehat{R}_{ \pm 1}\right)+S_{2}\left(\Psi, \widehat{R}_{ \pm 2}\right)\right)+\varepsilon \widehat{p}_{1} \\
\partial_{t} \widehat{R}_{-1} & =-i \omega_{1} \widehat{R}_{-1}-i \omega_{1}\left(S_{1}\left(\Psi, \widehat{R}_{ \pm 1}\right)+S_{2}\left(\Psi, \widehat{R}_{ \pm 2}\right)\right)+\varepsilon \widehat{p}_{-1} \\
\partial_{t} \widehat{R}_{2} & =i \omega_{2} \widehat{R}_{2}+i \omega_{2}^{-1}\left(S_{1}\left(\Psi, \widehat{R}_{ \pm 1}\right)+S_{2}\left(\Psi, \widehat{R}_{ \pm 2}\right)\right)+\varepsilon \widehat{p}_{2} \\
\partial_{t} \widehat{R}_{-2} & =-i \omega_{2} \widehat{R}_{-2}-i \omega_{2}^{-1}\left(S_{1}\left(\Psi, \widehat{R}_{ \pm 1}\right)+S_{2}\left(\Psi, \widehat{R}_{ \pm 2}\right)\right)+\varepsilon \widehat{p}_{-2}
\end{aligned}
$$

where $\Psi=\psi_{u}+\psi_{v}, S_{1}\left(\Psi, \widehat{R}_{ \pm 1}\right)=\widehat{\Psi} *\left(\widehat{R}_{1}+\widehat{R}_{-1}\right)$, and $S_{2}\left(\Psi, \widehat{R}_{ \pm 2}\right)=\widehat{\Psi} *\left(\widehat{R}_{2}+\widehat{R}_{-2}\right)$. The terms $\widehat{p}_{w}$ with $w \in\{ \pm 1, \pm 2\}$ can be estimated by

$$
\left\|\varepsilon \widehat{p}_{w}\right\|_{H_{s}^{0}} \leq C\left(\varepsilon^{3 / 2}\left(\left\|\widehat{R}_{-2}\right\|_{H_{s}^{0}}+\ldots+\left\|\widehat{R}_{2}\right\|_{H_{s}^{0}}\right)^{2}+\varepsilon\right)
$$




\subsection{The first normal form transformation}

From the term $S_{1}$ in the equation for $\widehat{R}_{ \pm 1}$ we already know from (1) that it can be estimated with the help of energy estimates. The main observation of [CS11] was that the long wave character w.r.t. time of the term $S_{2}$ in the equation for $\widehat{R}_{ \pm 2}$ can be used to construct an energy which allows to get rid of this term, too.

We try to eliminate the other terms with the help of normal form transformations. In order to do so we set $\widehat{R}_{l, 1}=\widehat{R}_{l}$ for $l \in\{ \pm 1, \pm 2\}$ and make the ansatz

$$
\begin{aligned}
\widehat{R}_{1,2} & =\widehat{R}_{1,1}+M_{1}^{(1)}\left(\Psi, \widehat{R}_{ \pm 2,1}\right) \\
\widehat{R}_{2,2} & =\widehat{R}_{2,1}+M_{2}^{(1)}\left(\Psi, \widehat{R}_{ \pm 1,1}\right) \\
\widehat{R}_{-1,2} & =\widehat{R}_{-1,1}+M_{-1}^{(1)}\left(\Psi, \widehat{R}_{ \pm 2,1}\right) \\
\widehat{R}_{-2,2} & =\widehat{R}_{-2,1}+M_{-2}^{(1)}\left(\Psi, \widehat{R}_{ \pm 1,1}\right)
\end{aligned}
$$

with $M_{1}^{(1)}$ and $M_{-1}^{(1)}$ linear in $\widehat{R}_{ \pm 2,1}$ as well as $M_{2}^{(1)}$ and $M_{-2}^{(1)}$ linear in $\widehat{R}_{ \pm 1,1}$, where $\widehat{R}_{ \pm n, j}=\left(\widehat{R}_{-n, j}, \widehat{R}_{n, j}\right)$.

Since $S_{2}$ is of the form

$$
S_{2}\left(\Psi, \widehat{R}_{ \pm 2,1}\right)=\sum_{l \in\{2,-2\}} S_{2 l}\left(\Psi, \widehat{R}_{l, 1}\right)
$$

with

$$
S_{2 l}\left(\Psi, \widehat{R}_{l, 1}\right)=\int \widehat{s}_{2 l}(k, k-m, m) \widehat{\Psi}(k-m, \varepsilon t) \widehat{R}_{l, 1}(m, t) d m,
$$

we set

$$
M_{1}^{(1)}\left(\Psi, \widehat{R}_{ \pm 2,1}\right)=\sum_{l \in\{2,-2\}} M_{1 l}^{(1)}\left(\Psi, \widehat{R}_{l, 1}\right)
$$

with

$$
M_{1 l}^{(1)}\left(\Psi, \widehat{R}_{l, 1}\right)=\int \widehat{m}_{1 l}^{(1)}(k, k-m, m) \widehat{\Psi}(k-m, \varepsilon t) \widehat{R}_{l, 1}(m, t) d m
$$


Using $\partial_{t} \Psi=\mathcal{O}(\varepsilon)$ we find

$$
\begin{aligned}
\partial_{t} \widehat{R}_{1,2}= & \partial_{t} \widehat{R}_{1,1}+\sum_{l \in\{2,-2\}} M_{1 l}^{(1)}\left(\Psi, \partial_{t} \widehat{R}_{l, 1}\right)+\mathcal{O}(\varepsilon) \\
= & i \omega_{1} \widehat{R}_{1,1}+i \omega_{1} S_{1}\left(\Psi, \widehat{R}_{ \pm 1,1}\right)+i \omega_{1} \sum_{l \in\{2,-2\}} S_{2 l}\left(\Psi, \widehat{R}_{l, 1}\right) \\
& +\sum_{l \in\{2,-2\}} M_{1 l}^{(1)}\left(\Psi, i \omega_{l} \widehat{R}_{l, 1}+i \omega_{l}^{-1}\left(S_{1}\left(\Psi, \widehat{R}_{ \pm 1,1}\right)+S_{2}\left(\Psi, \widehat{R}_{ \pm 2,1}\right)\right)\right) \\
& +\mathcal{O}(\varepsilon) \\
= & i \omega_{1} \widehat{R}_{1,2}-i \omega_{1} \sum_{l \in\{2,-2\}} M_{1 l}^{(1)}\left(\Psi, \widehat{R}_{l, 1}\right) \\
& +i \omega_{1} S_{1}\left(\Psi, \widehat{R}_{ \pm 1,1}\right)+i \omega_{1} \sum_{l \in\{2,-2\}} S_{2 l}\left(\Psi, \widehat{R}_{l, 1}\right) \\
& +\sum_{l \in\{2,-2\}} M_{1 l}^{(1)}\left(\Psi, i \omega_{l} \widehat{R}_{l, 1}+i \omega_{l}^{-1}\left(S_{1}\left(\Psi, \widehat{R}_{ \pm 1,1}\right)+S_{2}\left(\Psi, \widehat{R}_{ \pm 2,1}\right)\right)\right) \\
& +\mathcal{O}(\varepsilon) .
\end{aligned}
$$

In order to eliminate the term $S_{2 l}\left(\Psi, \widehat{R}_{l, 1}\right)$ we have to choose

$$
-i \omega_{1} M_{1 l}^{(1)}\left(\Psi, \widehat{R}_{l, 1}\right)+M_{1 l}^{(1)}\left(\Psi, i \omega_{l} \widehat{R}_{l, 1}\right)+i \omega_{1} S_{2 l}\left(\Psi, \widehat{R}_{l, 1}\right)=0
$$

and find

$$
i\left(\omega_{1}(k)-\omega_{l}(m)\right) \widehat{m}_{1 l}^{(1)}(k, k-m, m)=i \omega_{1}(k) \widehat{s}_{2 l}(k, k-m, m)
$$

for $l \in\{-2,2\}$. This equation can be solved w.r.t. the kernel $\widehat{m}_{1 l}^{(1)}(k, k-m, m)$ due to the validity of the non-resonance condition

$$
\inf _{k, l \in \mathbb{R}}\left|\omega_{1}(k) \pm \omega_{2}(m)\right| \geq 1
$$

Similarly the equation for $\widehat{R}_{2,2}$ can be handled. We find

$$
i\left(\omega_{2}(k)-\omega_{l}(m)\right) \widehat{m}_{2 l}^{(1)}(k, k-m, m)=i \omega_{2}^{-1}(k) \widehat{s}_{1 l}(k, k-m, m)
$$

for $l \in\{-1,1\}$, where $\widehat{s}_{1 l}$ is defined in an analogous way as $\widehat{s}_{2 l}$. This equation can be solved w.r.t. the kernel $\widehat{m}_{2 l}^{(1)}(k, k-m, m)$ due to the validity of the non-resonance condition

$$
\inf _{k, m \in \mathbb{R}}\left|\omega_{2}(k) \pm \omega_{1}(m)\right| \geq 1
$$


Although valid this non-resonance condition will be weakened in the following in order to present an approach which works for more general systems, too. After the transform we obtain

$$
\begin{aligned}
\partial_{t} \widehat{R}_{1,2}= & i \omega_{1} \widehat{R}_{1,2}+i \omega_{1} S_{1}\left(\Psi, \widehat{R}_{ \pm 1,1}\right) \\
& +\sum_{l \in\{2,-2\}} M_{1 l}^{(1)}\left(\Psi, i \omega_{l}^{-1}\left(S_{1}\left(\Psi, \widehat{R}_{ \pm 1,1}\right)+S_{2}\left(\Psi, \widehat{R}_{ \pm 2,1}\right)\right)\right)+\mathcal{O}(\varepsilon)
\end{aligned}
$$

and similarly for $\widehat{R}_{2,2}, \widehat{R}_{-1,2}$, and $\widehat{R}_{-2,2}$. On the right hand side there are still terms $\widehat{R}_{j, 1}$. In order to replace them with $\widehat{R}_{j, 2}$ terms we have to invert the above normal form transformation. We write the inverse as

$$
\begin{aligned}
& \widehat{R}_{ \pm 1,1}=\widehat{R}_{ \pm 1,2}+\widetilde{M}_{ \pm 1}^{(1)}\left(\Psi, \widehat{R}_{, 2}\right) \\
& \widehat{R}_{ \pm 2,1}=\widehat{R}_{ \pm 2,2}+\widetilde{M}_{ \pm 2}^{(1)}\left(\Psi, \widehat{R}_{, 2}\right)
\end{aligned}
$$

with $\widetilde{M}_{l}^{(1)}$ linear in $\widehat{R}_{, 2}$, where $\widehat{R}_{, j}=\left(\widehat{R}_{-2, j}, \widehat{R}_{-1, j}, \widehat{R}_{1, j}, \widehat{R}_{2, j}\right)$. Substituting $\widehat{R}_{, 1}$ in terms of $\widehat{R}_{, 2}$ finally yields

$$
\begin{aligned}
\partial_{t} \widehat{R}_{1,2}= & i \omega_{1} \widehat{R}_{1,2}+i \omega_{1} S_{1}\left(\Psi, \widehat{R}_{ \pm 1,2}\right)+i \omega_{1} S_{1}\left(\Psi, \widetilde{M}_{ \pm 1}^{(1)}\left(\Psi, \widehat{R}_{, 2}\right)\right) \\
& +\sum_{l \in\{2,-2\}}\left(M_{1 l}^{(1)}\left(\Psi, i \omega_{l}^{-1} S_{1}\left(\Psi, \widehat{R}_{ \pm 1,2}\right)\right)+M_{1 l}^{(1)}\left(\Psi, i \omega_{l}^{-1} S_{1}\left(\Psi, \widetilde{M}_{ \pm 1}^{(1)}\left(\Psi, \widehat{R}_{, 2}\right)\right)\right)\right) \\
& +\sum_{l \in\{2,-2\}}\left(M_{1 l}^{(1)}\left(\Psi, i \omega_{l}^{-1} S_{2}\left(\Psi, \widehat{R}_{ \pm 2,2}\right)\right)+M_{1 l}^{(1)}\left(\Psi, i \omega_{l}^{-1} S_{2}\left(\Psi, \widetilde{M}_{ \pm 2}^{(1)}\left(\Psi, \widehat{R}_{, 2}\right)\right)\right)\right) \\
& +\mathcal{O}(\varepsilon)
\end{aligned}
$$

and similarly for $\widehat{R}_{2,2}, \widehat{R}_{-1,2}$, and $\widehat{R}_{-2,2}$. Hence new terms of order $\mathcal{O}(1)$ are created. However, there is only one term of order $\mathcal{O}(\|\Psi\|)$, namely $i \omega_{1} S_{1}\left(\Psi, \widehat{R}_{ \pm 1,2}\right)$ from which we already know how to handle it via (1) with the help of energy estimates. All other terms are of order $\mathcal{O}\left(\|\Psi\|^{2}\right)$ or higher. Some of them are resonant, but of long wave form and will be included into the energy estimates.

Some of them are non-resonant but not of long wave form. They will be eliminated by another normal form transform, but ad infinitum by such transformations terms of order $\mathcal{O}(1)$ are created. Therefore, we have to prove the convergence of this procedure. It is based on the fact that in the $j$-th step only terms of order $\mathcal{O}\left(\|\Psi\|^{j}\right)$ or higher will be affected. This will be discussed in detail in the subsequent sections. 


\subsection{The recursion formulas}

In order to control the action of the infinitely many near-identity changes of variables which we will apply to (21)-(24) it is essential to extract the structure of this system. We will see that after performing $j-1$ transformations the error equations will be of the form

$$
\begin{aligned}
\partial_{t} \widehat{R}_{1, j}(k, t)= & i \omega_{1}(k) \widehat{R}_{1, j}(k, t)+\varepsilon \widehat{p}_{1, j}(k, t) \\
& +i \omega_{1}(k) \int \widehat{f}_{1, r e s}^{(j)}(k, k-m, \varepsilon t)\left(\widehat{R}_{1, j}(m, t)+\widehat{R}_{-1, j}(m, t)\right) d m \\
& +i \omega_{1}(k) \int \widehat{f}_{1, \text { non }}^{(j)}(k, k-m, \varepsilon t)\left(\widehat{R}_{2, j}(m, t)+\widehat{R}_{-2, j}(m, t)\right) d m \\
\partial_{t} \widehat{R}_{2, j}(k, t)= & i \omega_{2}(k) \widehat{R}_{2, j}(k, t)+\varepsilon \widehat{p}_{2, j}(k, t) \\
& +i \omega_{2}^{-1}(k) \int \widehat{f}_{2, \text { non }}^{(j)}(k, k-m, \varepsilon t)\left(\widehat{R}_{1, j}(m, t)+\widehat{R}_{-1, j}(m, t)\right) d m \\
& +i \omega_{2}^{-1}(k) \int \widehat{f}_{2, r e s}^{(j)}(k, k-m, \varepsilon t)\left(\widehat{R}_{2 j}(m, t)+\widehat{R}_{-2, j}(m, t)\right) d m
\end{aligned}
$$

and similarly for $\partial_{t} \widehat{R}_{-1, j}$ and $\partial_{t} \widehat{R}_{-2, j}$. For $l \in\{ \pm 1, \pm 2\}$ we set $\widehat{p}_{l, 1}=\widehat{p}_{l}$ and find

$$
\widehat{f}_{l, r e s}^{(1)}(k, k-m, \varepsilon t)=\widehat{f}_{l, n o n}^{(1)}(k, k-m, \varepsilon t)=\widehat{\Psi}(k-m, \varepsilon t) .
$$

Since $\widehat{R}_{-l, j}$ will be the complex conjugate of $\widehat{R}_{l, j}$, we have

$$
\widehat{f}_{-l, r e s}^{(j)}=\overline{\widehat{f}_{l, r e s}^{(j)}}, \quad \widehat{f}_{-l, n o n}^{(j)}=\overline{\widehat{f}_{l, n o n}^{(j)}}, \quad \text { and } \quad \widehat{p}_{-l, j}=\overline{\widehat{p}_{l, j}}
$$

for $j \in \mathbb{N}$ and $l \in\{1,2\}$. Hence, it is sufficient to analyze the equations for $\widehat{R}_{|l|, j}$.

We derive now recursion formulas for the terms $\widehat{p}_{l, j}, \widehat{f}_{l, \text { res }}^{(j)}$, and $\widehat{f}_{l, \text { non }}^{(j)}$. In order to do so we introduce the $j$-th near identity change of variables by

$$
\begin{aligned}
\widehat{R}_{1, j+1}(k, t) & =\widehat{R}_{1, j}(k, t)+\sum_{l \in\{2,-2\}} \int \widehat{g}_{1 l}^{(j)}(k, k-m, \varepsilon t) \widehat{R}_{l, j}(m, t) d m, \\
\widehat{R}_{2, j+1}(k, t) & =\widehat{R}_{2, j}(k, t)+\sum_{l \in\{1,-1\}} \int \widehat{g}_{2 l}^{(j)}(k, k-m, \varepsilon t) \widehat{R}_{l, j}(m, t) d m .
\end{aligned}
$$

The perturbation of the identity is chosen in accordance with the validity of the non-resonance condition 10 . We assume for the moment that the transformation 
(35)-(36) is invertible and that its inverse has the form

$$
\widehat{R}_{i, j}(k, t)=\widehat{R}_{i, j+1}(k, t)+\sum_{l \in\{ \pm 1, \pm 2\}} \int \widehat{h}_{i l}^{(j)}(k, k-m, \varepsilon t) \widehat{R}_{l, j+1}(m, t) d m
$$

for $i \in\{ \pm 1, \pm 2\}$. Differentiating (35) w.r.t. time yields

$$
\begin{aligned}
\partial_{t} \widehat{R}_{1, j+1}(k, t)= & \partial_{t} \widehat{R}_{1, j}(k, t)+\sum_{l \in\{2,-2\}} \int \widehat{g}_{1 l}^{(j)}(k, k-m, \varepsilon t) \partial_{t} \widehat{R}_{l, j}(m, t) d m \\
& +\varepsilon \sum_{l \in\{2,-2\}} \int \partial_{T} \widehat{g}_{1 l}^{(j)}(k, k-m, \varepsilon t) \widehat{R}_{l, j}(m, t) d m .
\end{aligned}
$$

Then we replace the $\partial_{t} \widehat{R}_{l, j}(k, t)$ by the equations $\left.32-33\right)$. Finally, we replace the $\widehat{R}_{l, j}(k, t)$ via (37) by the $\widehat{R}_{l, j+1}(k, t)$. In order to eliminate for $l \in\{ \pm 2\}$ the nonresonant term

$$
i \omega_{1}(k) \int \widehat{f}_{1, n o n}^{(j)}(k, k-m, \varepsilon t) \widehat{R}_{l, j}(m, t) d m
$$

we could proceed as above and choose the functions $\widehat{g}_{1 l}^{(j)}$ to satisfy

$$
\begin{aligned}
0= & -i \omega_{1}(k) \int \widehat{g}_{1 l}^{(j)}(k, k-m, \varepsilon t) \widehat{R}_{l, j}(m, t) d m \\
& +\int i \omega_{l}(m) \widehat{g}_{1 l}^{(j)}(k, k-m, \varepsilon t) \widehat{R}_{l, j}(m, t) d m \\
& +i \omega_{1}(k) \int \widehat{f}_{1, n o n}^{(j)}(k, k-m, \varepsilon t) \widehat{R}_{l, j}(m, t) d m
\end{aligned}
$$

or equivalently to satisfy

$$
\widehat{g}_{1 l}^{(j)}(k, k-m, \varepsilon t)=i \omega_{1}(k)\left(i \omega_{1}(k)-i \omega_{l}(m)\right)^{-1} \widehat{f}_{1, n o n}^{(j)}(k, k-m, \varepsilon t) .
$$

However, for more general systems the non-resonance condition 29 will not be valid. Hence, in order to present an approach which works for more general systems, too, we proceed differently at this point. Since $\widehat{\Psi}$ is strongly concentrated at the wave number $k=0$ the difference

$$
\varepsilon \widehat{r}_{1, j}(k, t)=\sum_{l \in\{2,-2\}} \int i\left(\omega_{l}(m)-\omega_{l}(k)\right) \widehat{g}_{1 l}^{(j)}(k, k-m, \varepsilon t) \widehat{R}_{l, j}(m, t) d m
$$


will be of order $\mathcal{O}(\varepsilon)$. This can be expected for $j=1$ due to (34) and will be proved subsequently by induction for all $j \geq 1$. By replacing (38) by

$$
\begin{aligned}
0= & -i \omega_{1}(k) \int \widehat{g}_{1 l}^{(j)}(k, k-m, \varepsilon t) \widehat{R}_{l, j}(m, t) d m \\
& +\int i \omega_{l}(k) \widehat{g}_{1 l}^{(j)}(k, k-m, \varepsilon t) \widehat{R}_{l, j}(m, t) d m \\
& +i \omega_{1}(k) \int \widehat{f}_{1, n o n}^{(j)}(k, k-m, \varepsilon t) \widehat{R}_{l, j}(m, t) d m
\end{aligned}
$$

or equivalently

$$
\widehat{g}_{1 l}^{(j)}(k, k-m, \varepsilon t)=i \omega_{1}(k)\left(i \omega_{1}(k)-i \omega_{l}(k)\right)^{-1} \widehat{f}_{1, n o n}^{(j)}(k, k-m, \varepsilon t) .
$$

we only made an error of order $\mathcal{O}(\varepsilon)$ and come to the much weaker non-resonance condition 10p.

By a straightforward calculation we find

$$
\begin{aligned}
& \widehat{f}_{1, \text { res }}^{(j+1)}(k, k-m, \varepsilon t)-\widehat{f}_{1, \text { res }}^{(j)}(k, k-m, \varepsilon t) \\
= & \sum_{\lambda \in\{2,-2\}} \int \widehat{\widetilde{g}}_{1 \lambda}^{(j)}(k, k-l, \varepsilon t) \omega_{2}^{-1}(l) \widehat{f}_{\lambda, \text { non }}^{(j)}(l, l-m, \varepsilon t) d l \\
& +\sum_{\kappa \in\{1,-1\}} \int \widehat{f}_{1, r e s}^{(j)}(k, k-l, \varepsilon t) \widehat{h}_{\kappa \mu}^{(j)}(l, l-m, \varepsilon t) d l \\
& +\sum_{\substack{\lambda \in\{2,-2\}, \kappa \in\{1,-1\}}} \iint \widehat{\widetilde{g}}_{1 \lambda}^{(j)}\left(k, k-l_{1}, \varepsilon t\right) \omega_{2}^{-1}\left(l_{1}\right) \widehat{f}_{\lambda, \text { non }}^{(j)}\left(l_{1}, l_{1}-l_{2}, \varepsilon t\right) \widehat{h}_{\kappa \mu}^{(j)}\left(l_{2}, l_{2}-m, \varepsilon t\right) d l_{2} d l_{1} \\
& +\sum_{\substack{\lambda \in\{2,-2\}, \kappa \in\{2,-2\}}} \iint \widehat{\widehat{g}}_{1 \lambda}^{(j)}\left(k, k-l_{1}, \varepsilon t\right) \omega_{2}^{-1}\left(l_{1}\right) \widehat{f}_{\lambda, r e s}^{(j)}\left(l_{1}, l_{1}-l_{2}, \varepsilon t\right) \widehat{h}_{\kappa \mu}^{(j)}\left(l_{2}, l_{2}-m, \varepsilon t\right) d l_{2} d l_{1},
\end{aligned}
$$


and

$$
\begin{aligned}
& \widehat{f}_{1, \text { non }}^{(j+1)}(k, k-m, \varepsilon t) \\
= & \sum_{\lambda \in\{2,-2\}} \int \widehat{\widetilde{g}}_{1 \lambda}^{(j)}(k, k-l, \varepsilon t) \omega_{2}^{-1}(l) \widehat{f}_{\lambda, r e s}^{(j)}(l, l-m, \varepsilon t) d l \\
& +\sum_{\kappa \in\{1,-1\}} \int \widehat{f}_{1, r e s}^{(j)}(k, k-l, \varepsilon t) \widehat{h}_{\kappa \mu}^{(j)}(l, l-m, \varepsilon t) d l \\
& +\sum_{\substack{\lambda \in\{2,-2\} \\
\kappa \in\{1,-1\}}} \iint \widehat{\widetilde{g}}_{1 \lambda}^{(j)}\left(k, k-l_{1}, \varepsilon t\right) \omega_{2}^{-1}\left(l_{1}\right) \widehat{f}_{\lambda, \text { non }}^{(j)}\left(l_{1}, l_{1}-l_{2}, \varepsilon t\right) \widehat{h}_{\kappa \mu}^{(j)}\left(l_{2}, l_{2}-m, \varepsilon t\right) d l_{2} d l_{1} \\
& +\sum_{\substack{\lambda \in\{2,-2\}, \kappa \in\{2,-2\}}} \iint \widehat{\widetilde{g}}_{1 \lambda}^{(j)}\left(k, k-l_{1}, \varepsilon t\right) \omega_{2}^{-1}\left(l_{1}\right) \widehat{f}_{\lambda, r e s}^{(j)}\left(l_{1}, l_{1}-l_{2}, \varepsilon t\right) \widehat{h}_{\kappa \mu}^{(j)}\left(l_{2}, l_{2}-m, \varepsilon t\right) d l_{2} d l_{1},
\end{aligned}
$$

where we used the abbreviation

$\widehat{\widetilde{g}}_{1 \lambda}^{(j)}(k, k-l, \varepsilon t)=\left(\omega_{1}(k)\right)^{-1} \widehat{g}_{1 \lambda}^{(j)}(k, k-l, \varepsilon t)=-i\left(i \omega_{1}(k)-i \omega_{\lambda}(k)\right)^{-1} \widehat{f}_{1, \text { non }}^{(j)}(k, k-m, \varepsilon t)$.

Moreover, we have

$$
\begin{aligned}
\widehat{p}_{1, j+1}(k, t)-\widehat{p}_{1, j}(k, t)= & \sum_{\lambda \in\{2,-2\}} \int \partial_{T} \widehat{g}_{1 \lambda}^{(j)}(k, k-m, \varepsilon t) \widehat{R}_{\lambda, j}(m, t) d m \\
& +\sum_{\lambda \in\{2,-2\}} \int \widehat{g}_{1 \lambda}^{(j)}(k, k-m, \varepsilon t) \widehat{p}_{\lambda, j}(m, t) d m+\widehat{r}_{1, j}(k, t) .
\end{aligned}
$$

Due to the symmetry in $(32)$ and $(33)$ we obtain similar equations for $\widehat{R}_{2}$ but with the roles of $\widehat{R}_{2}$ and $\widehat{R}_{1}$ interchanged.

\subsection{The functional analytic set-up and the inversion of the normal form transformations}

In order to control the functions $f, g$, and $h$, we introduce the norm

$$
\|f\|_{X^{s, \varepsilon}}:=\int \sup _{k \in \mathbb{R}}|f(k, l)|\left(1+(l / \varepsilon)^{2}\right)^{s / 2} d l .
$$

This norm reflects that $f, g$, and $h$ are (infinite) sums of terms $\widehat{\kappa}^{(j)}(k) \varepsilon^{-1} \widehat{\varphi}^{(j)}\left(\frac{k-m}{\varepsilon}, \varepsilon t\right)$, where $\widehat{\kappa}^{(j)}$ is Lipschitz continuous and determined by $\omega_{1}, \omega_{2}$ and where $\widehat{\varphi}^{(j)}(\cdot, \varepsilon t)$ belongs to $L_{s+1}^{1}$ and is determined by $\widehat{\Psi}(\cdot, \varepsilon t)$. With the help of Young's inequality for 
convolutions we have

$$
\left\|\int \widehat{f}(k, k-m, \varepsilon t) \widehat{R}(m, t) d m\right\|_{H_{s}^{0}} \leq C\|\widehat{f}\|_{X^{s, \varepsilon}}\|\widehat{R}\|_{H_{s}^{0}}
$$

The following lemma allows us to control the convolution of $f, g$, and $h$ in the previous recursion formulas.

Lemma 3.1. For $s>0$ the following estimate holds:

$$
\left\|\int_{\mathbb{R}} f\left(\cdot \cdot_{1}, \cdot 1-l\right) g\left(l, l-\cdot_{2}\right) d l\right\|_{X^{s, \varepsilon}} \leq\|f\|_{X^{s, \varepsilon}}\|g\|_{X^{s, \varepsilon}}
$$

Proof. Using Young's inequality for convolutions in weighted $L^{1}$-spaces yields

$$
\begin{aligned}
& \int \sup _{k \in \mathbb{R}}\left|\int f(k, k-l) g(l, l-m) d l\right|\left(1+((k-m) / \varepsilon)^{2}\right)^{s / 2} d(k-m) \\
& \leq \int \sup _{k \in \mathbb{R}} \int \sup _{\widetilde{k} \in \mathbb{R}}|f(\widetilde{k}, k-l)| \sup _{\widetilde{k} \in \mathbb{R}}|g(\widetilde{k}, l-k+m)| d l\left(1+(m / \varepsilon)^{2}\right)^{s / 2} d m \\
& \leq \int \sup _{k \in \mathbb{R}} \int \sup _{\widetilde{k} \in \mathbb{R}}|f(\widetilde{k}, l)| \sup _{\widetilde{k} \in \mathbb{R}}|g(\widetilde{k}, m-l)| d l\left(1+(m / \varepsilon)^{2}\right)^{s / 2} d m \\
& \leq C \int \sup _{k \in \mathbb{R}}|f(k, l)|\left(1+(l / \varepsilon)^{2}\right)^{s / 2} d l \int \sup _{k \in \mathbb{R}}|g(k, m)|\left(1+(m / \varepsilon)^{2}\right)^{s / 2} d m \\
& =\|f(k, l)\|_{X^{s, \varepsilon}}\|g(k, l)\|_{X^{s, \varepsilon}} .
\end{aligned}
$$

For $\left\|\widehat{g}_{i l}^{(j)}(\varepsilon t)\right\|_{X^{s, \varepsilon}}$ sufficiently small, but independent of $0<\varepsilon \ll 1$, the transformation (35)-(36) is invertible.

Lemma 3.2. For $\widehat{R}_{i, j}(t) \in H_{s}^{0}$ with $s \geq 1$ define $\widehat{R}_{i, j+1}(t)$ by

$$
\widehat{R}_{j+1}(k, t)=\left(I+T^{(j)}\right)\left(\widehat{R}_{j}(k, t)\right),
$$

where

$$
T^{(j)}\left(\widehat{R}_{j}\right)=\left(\begin{array}{cccc}
0 & 0 & T_{12}^{(j)} & T_{1-2}^{(j)} \\
0 & 0 & T_{-12}^{(j)} & T_{-1-2}^{(j)} \\
T_{21}^{(j)} & T_{2-1}^{(j)} & 0 & 0 \\
T_{-21}^{(j)} & T_{-2-1}^{(j)} & 0 & 0
\end{array}\right)\left(\begin{array}{c}
\widehat{R}_{1, j} \\
\widehat{R}_{-1, j} \\
\widehat{R}_{2, j} \\
\widehat{R}_{-2, j}
\end{array}\right)
$$


with

$$
\left(T_{i l}^{(j)} \widehat{R}_{l, j}\right)(k, t)=\int \widehat{g}_{i l}^{(j)}(k, k-m, \varepsilon t) \widehat{R}_{l, j}(m, t) d m .
$$

Then there exists a $q>0$ such that if

$$
\left\|\widehat{g}_{i l}^{(j)}(\varepsilon t)\right\|_{X^{s, \varepsilon}} \leq q
$$

holds for all $i, l \in\{ \pm 1, \pm 2\}$, the transformation (46) is bijective and has an inverse of the form

$$
\widehat{R}_{i, j}(k, t)=\widehat{R}_{i, j+1}(k, t)+\sum_{l \in\{1,-1,2,-2\}} \int \widehat{h}_{i l}^{(j)}(k, k-m, \varepsilon t) \widehat{R}_{l, j+1}(m, t) d m
$$

with

$$
\left\|\widehat{h}^{(j)}(\varepsilon t)\right\|_{X^{s, \varepsilon}} \leq \frac{C\left\|\widehat{g}^{(j)}(\varepsilon t)\right\|_{X^{s, \varepsilon}}}{1-\left\|\widehat{g}^{(j)}(\varepsilon t)\right\|_{X^{s, \varepsilon}}}
$$

where $\left\|\widehat{g}^{(j)}(\varepsilon t)\right\|_{X^{s, \varepsilon}}=\max _{i, l \in\{ \pm 1, \pm 2\}}\left\{\left\|\widehat{g}_{i l}^{(j)}(\varepsilon t)\right\|_{X^{s, \varepsilon}}\right\}$.

Proof. Let $t$ be fixed. Obviously $T^{(j)}:\left(H_{s}^{0}\right)^{4} \rightarrow\left(H_{s}^{0}\right)^{4}$ is a linear operator. Let $T_{1}^{(j)}$ and $T_{2}^{(j)}$ be defined as

$$
T_{1}^{(j)}=\left(\begin{array}{cc}
T_{12}^{(j)} & T_{1-2}^{(j)} \\
T_{-12}^{(j)} & T_{-1-2}^{(j)}
\end{array}\right) \quad \text { and } \quad T_{2}^{(j)}=\left(\begin{array}{cc}
T_{21}^{(j)} & T_{2-1}^{(j)} \\
T_{-21}^{(j)} & T_{-2-1}^{(j)}
\end{array}\right) .
$$

We set $\left\|T^{(j)}\right\|:=\max \left\{\left\|T_{1}^{(j)}\right\|,\left\|T_{2}^{(j)}\right\|\right\}$ with

$$
\left\|T_{1}^{(j)}\right\|^{2}=\sup _{\left\|\left(\widehat{R}_{2, j}, \widehat{R}_{-2, j}\right)^{T}\right\| \leq 1}\left(\sum_{l \in\{2,-2\}}\left\|T_{1 l}^{(j)}\left(\widehat{R}_{l, j}\right)\right\|_{H_{s}^{0}}^{2}+\sum_{l \in\{2,-2\}}\left\|T_{-1 l}^{(j)}\left(\widehat{R}_{l, j}\right)\right\|_{H_{s}^{0}}^{2}\right) .
$$

By (45) we have

$$
\left\|T_{i l}^{(j)} \widehat{R}_{l, j}\right\|_{H_{s}^{0}} \leq C\left\|\widehat{g}_{i l}^{(j)}\right\|_{X^{s, \varepsilon}}\left\|\widehat{R}_{r, j}\right\|_{H_{s}^{0}} .
$$

Hence we have $\left\|T^{(j)}\right\|=\mathcal{O}(q)$. Therefore, for $q>0$ sufficiently small we can use Neumann's series to invert

$$
\left(I-\left(-T^{(j)}\right)\right)^{-1}=\sum_{\lambda=0}^{\infty}\left(-T^{(j)}\right)^{\circ \lambda}
$$


where we denoted by $\left(T^{(j)}\right)^{\circ \lambda}$ the $\lambda$-times composition of $T^{(j)}$. Thus we obtain a composition of operators as in Lemma 3.2 . For each pair $T_{i l}^{(j)}$ and $T_{s t}^{(j)}$ we can write

$$
\begin{aligned}
\left(T_{i l}^{(j)} \circ T_{s t}^{(j)}\right) \widehat{R}_{1, j+1} & =\int \widehat{g}_{i l}^{(j)}(k, k-m) \int \widehat{g}_{s t}^{(j)}(m, m-n) \widehat{R}_{1, j+1}(n) d n d m \\
& =\iint \widehat{g}_{i l}^{(j)}(k, k-m) g_{s t}^{(j)}(m, m-n) d m \widehat{R}_{1, j+1}(n) d n .
\end{aligned}
$$

Hence we obtain inductively a series of integral kernels as in (37). The $X^{s, \varepsilon}$-norm of $\widehat{h}_{i k}^{(j)}$ is bounded by

$$
\left\|h^{(j)}\right\|_{X^{s, \varepsilon}} \leq C \sum_{l=1}^{\infty}\left(\left\|g^{(j)}\right\|\right)^{l}=\frac{C\left\|\widehat{g}^{(j)}\right\|_{X^{s, \varepsilon}}}{1-\left\|\widehat{g}^{(j)}\right\|_{X^{s, \varepsilon}}} .
$$

This is exactly 49 .

\subsection{Proof of convergence}

In Lemma 3.2. we assumed that (48) holds. Here we wish to show that all the $\hat{f}_{.}^{(j)}$, $\hat{g}_{\cdot}^{(j)}$ and $\hat{h}_{\cdot}^{(j)}$ do in fact satisfy such estimates or even sharper estimates. A simple application of Lemma 3.1 to (41) and 42 gives the estimates

$$
\begin{aligned}
\left\|\widehat{f}_{1, r e s}^{(j+1)}-\widehat{f}_{1, r e s}^{(j)}\right\|_{X^{s, \varepsilon}} \leq & \sum_{\lambda \in\{2,-2\}}\left\|\widehat{\widetilde{g}}_{1 \lambda}^{(j)}\right\|_{X^{s, \varepsilon}}\left\|\widehat{f}_{\lambda, n o n}^{(j)}\right\|_{X^{s, \varepsilon}}+\left\|\widehat{f}_{1, r e s}^{(j)}\right\|_{X^{s, \varepsilon}} \sum_{\kappa \in\{1,-1\}}\left\|\widehat{h}_{\kappa \mu}^{(j)}\right\|_{X^{s, \varepsilon}} \\
& +\sum_{\lambda \in\{2,-2\}}\left\|\widehat{\widetilde{g}}_{1 \lambda}^{(j)}\right\|_{X^{s, \varepsilon}}\left\|\widehat{f}_{\lambda, n o n}^{(j)}\right\|_{X^{s, \varepsilon}} \sum_{\kappa \in\{1,-1\}}\left\|\widehat{h}_{\kappa \mu}^{(j)}\right\|_{X^{s, \varepsilon}} \\
& +\sum_{\lambda \in\{2,-2\}}\left\|\widehat{\widetilde{g}}_{1 \lambda}^{(j)}\right\|_{X^{s, \varepsilon}}\left\|\widehat{f}_{\lambda, r e s}^{(j)}\right\|_{X^{s, \varepsilon}} \sum_{\kappa \in\{2,-2\}}\left\|\widehat{h}_{\kappa \mu}^{(j)}\right\|_{X^{s, \varepsilon}}
\end{aligned}
$$

and

$$
\begin{aligned}
\left\|\widehat{f}_{1, n o n}^{(j+1)}\right\|_{X^{s, \varepsilon}} \leq & \sum_{\lambda \in\{2,-2\}}\left\|\widehat{\widetilde{g}}_{1 \lambda}^{(j)}\right\|_{X^{s, \varepsilon}}\left\|\widehat{f}_{\lambda, r e s}^{(j)}\right\|_{X^{s, \varepsilon}}+\left\|\widehat{f}_{1, r e s}^{(j)}\right\|_{X^{s, \varepsilon}} \sum_{\kappa \in\{1,-1\}}\left\|\widehat{h}_{\kappa \mu}^{(j)}\right\|_{X^{s, \varepsilon}} \\
& +\sum_{\lambda \in\{2,-2\}}\left\|\widehat{\widetilde{g}}_{1 \lambda}^{(j)}\right\|_{X^{s, \varepsilon}}\left\|\widehat{f}_{\lambda, n o n}^{(j)}\right\|_{X^{s, \varepsilon}} \sum_{\kappa \in\{1,-1\}}\left\|\widehat{h}_{\kappa \mu}^{(j)}\right\|_{X^{s, \varepsilon}} \\
& +\sum_{\lambda \in\{2,-2\}}\left\|\widehat{\widetilde{g}}_{1 \lambda}^{(j)}\right\|_{X^{s, \varepsilon}}\left\|\widehat{f}_{\lambda, r e s}^{(j)}\right\|_{X^{s, \varepsilon}} \sum_{\kappa \in\{2,-2\}}\left\|\widehat{h}_{\kappa \mu}^{(j)}\right\|_{X^{s, \varepsilon}}
\end{aligned}
$$


One can obtain analogous inequalities for $\left\|\widehat{f}_{2, \text { res }}^{(j+1)}-\widehat{f}_{2, \text { res }}^{(j)}\right\|_{X^{s, \varepsilon}}$ and $\left\|\widehat{f}_{2, \text { non }}^{(j+1)}\right\|_{X^{s, \varepsilon}}$ with suitably adjusted indices. We will now give the important estimates mentioned earlier, from which our main convergence results will follow.

Lemma 3.3. There exists a $q>0$ such that for

$$
\left\|\widehat{f}_{\nu, r e s}^{(1)}\right\|_{X^{s, \varepsilon}}+\left\|\widehat{f}_{\nu, n o n}^{(1)}\right\|_{X^{s, \varepsilon}} \leq q, \quad \nu \in\{1,-1,2,-2\}
$$

we have
a) $\left\|\widehat{f}_{\kappa, r e s}^{(j)}\right\|_{X^{s, \varepsilon}} \leq q \frac{1-q^{\frac{j}{2}}}{1-q^{\frac{1}{2}}}$
b) $\left\|\widehat{f}_{\kappa, n o n}^{(j)}\right\|_{X^{s, \varepsilon}} \leq q^{\frac{j+1}{2}}$,
c) $\left\|\widehat{g}_{\kappa \lambda}^{(j)}\right\|_{X^{s, \varepsilon}} \leq C_{\omega} q^{\frac{j+1}{2}}$,
d) $\left\|\widehat{h}_{\kappa \lambda}^{(j)}\right\|_{X^{s, \varepsilon}} \leq 2 C_{\omega} q^{\frac{j+1}{2}}$
e) $\left\|\widehat{f}_{\kappa, r e s}^{(j+1)}-\widehat{f}_{\kappa, r e s}^{(j)}\right\|_{X^{s, \varepsilon}} \leq q q^{\frac{j}{2}}$

for all $j \in \mathbb{N}$ and $\kappa, \lambda \in\{2,-2,1,-1\}$, where

$$
C_{\omega}=\max _{\mu \in\{1,-1\}} \sup _{\lambda \in\{2,-2\}}\left|i \omega_{\mu}(k)-i \omega_{\lambda}(k)\right|^{-1} .
$$

Proof. The proof is based on an induction argument.

i) For $j=1$ the estimates a) and b) follow from (53) and the assertion in c) follows from (40). From (49) we have

$$
\left\|h_{i k}^{(j)}\right\|_{X^{s, \varepsilon}} \leq \frac{\left\|\widehat{g}^{(j)}\right\|_{X^{s, \varepsilon}}}{1-\left\|\widehat{g}^{(j)}\right\|_{X^{s, \varepsilon}}}
$$

If $q$ is chosen smaller than $\frac{1}{2 C_{\omega}}$, then due to the induction basis for c) we obtain the assertion in $\mathrm{d}$ ) for $j=1$.

ii) Using (40), (49) and (51) we obtain with the abbreviation $\left\|\widehat{f}_{n}^{(j)}\right\|_{X^{s, \varepsilon}}:=$ $\max _{\kappa \in\{1,-1,2,-2\}}\left\|\widehat{f}_{\kappa, \text { non }}^{(j)}\right\|_{X^{s, \varepsilon}}$ that

$$
\begin{aligned}
\left\|\widehat{f}_{1, \text { res }}^{(j+1)}-\widehat{f}_{1, \text { res }}^{(j)}\right\|_{X^{s, \varepsilon}} \leq \quad & 2 C_{\omega}\left\|\widehat{f}_{, \text {non }}^{(j)}\right\|_{X^{s, \varepsilon}}^{2}+4 C_{\omega}\left\|\widehat{f}_{1, r e s}^{(j)}\right\|_{X^{s, \varepsilon}}\left\|\widehat{f}_{, \text {non }}^{(j)}\right\|_{X^{s, \varepsilon}} \\
& +8 C_{\omega}^{2}\left\|\widehat{f}_{, \text {non }}^{(j)}\right\|_{X^{s, \varepsilon}}^{3}+8 C_{\omega}^{2}\left\|\widehat{f}_{, \text {non }}^{(j)}\right\|_{X^{s, \varepsilon}}^{2}\left\|\widehat{f}_{2, \text { res }}^{(j)}\right\|_{X^{s, \varepsilon}}
\end{aligned}
$$

Using the induction hypotheses $\left\|\widehat{f}_{i, n o n}^{(j)}\right\|_{X^{s, \varepsilon}} \leq q^{\frac{j+1}{2}}$ and $\left\|\widehat{f}_{i, r e s}^{(j)}\right\|_{X^{s, \varepsilon}} \leq q \frac{1-q^{\frac{j}{2}}}{1-q^{\frac{1}{2}}}$ we find

$$
\left\|\widehat{f}_{1, \text { res }}^{(j+1)}-\widehat{f}_{1, \text { res }}^{(j)}\right\|_{X^{s, \varepsilon}} \leq 2 C_{\omega} q^{j+1}+\frac{4 C_{\omega}}{1-q^{\frac{1}{2}}} q^{\frac{j+3}{2}}+8 C_{\omega}^{2} q^{\frac{3(j+1)}{2}}+\frac{8 C_{\omega}^{2}}{1-q^{\frac{1}{2}}} q^{j+2} \leq q q^{\frac{j}{2}}
$$


which implies e) for $q>0$ sufficiently small. Since $\widehat{f}_{1, \text { res }}^{(j+1)}=\widehat{f}_{1, \text { res }}^{(1)}+\sum_{k=1}^{j}\left(\widehat{f}_{1, \text { res }}^{(k+1)}-\right.$ $\left.\widehat{f}_{1, \text { res }}^{(k)}\right)$ we estimate

$$
\left\|\widehat{f}_{1, r e s}^{(j+1)}\right\|_{X^{s, \varepsilon}} \leq q \sum_{k=0}^{j} q^{\frac{k}{2}}=q \frac{1-q^{\frac{j+1}{2}}}{1-q^{\frac{1}{2}}}
$$

Using (52) and (49) we estimate $\left\|\widehat{f}_{i, n o n}^{(j)}\right\|_{X^{s, \varepsilon}}$ similarly to the resonant terms by

$$
\left\|\widehat{f}_{i, \text { non }}^{(j+1)}\right\|_{X^{s, \varepsilon}} \leq 6 C_{\omega} q^{\frac{j+1}{2}} q \frac{1-q^{\frac{j}{2}}}{1-q^{\frac{1}{2}}}+8 C_{\omega} q^{\frac{3(j+1)}{2}}+8 C_{\omega} q^{j+2} \frac{1-q^{\frac{j}{2}}}{1-q^{\frac{1}{2}}} \leq q^{\frac{j+2}{2}}
$$

for $q>0$ sufficiently small. With the help of (49) we obtain the estimates c) and d).

Remark 3.4. Due to (34) we have for $\|\widehat{\Psi}\|_{L_{s}^{1}}$ sufficiently small that $\left\|\widehat{f}_{i, r e s}^{(1)}\right\|_{X^{s, \varepsilon}}+$ $\left\|\widehat{f}_{i, n o n}^{(1)}\right\|_{X^{s, \varepsilon}} \leq q$ for a $0<q \ll 1$ independent of $0<\varepsilon \ll 1$.

Remark 3.5. Similar estimates as in Lemma 3.3 hold for the partial derivatives of $\widehat{f}_{i, r e s}^{(j)}(k, l, \varepsilon t), \widehat{g}_{i r}^{(j)}(k, l, \varepsilon t)$ and $\widehat{h}_{i r}^{(j)}(k, l, \varepsilon t)$ w.r.t. the first and the third argument, because the functions $\omega_{\lambda}(k)$ with $\lambda \in\{ \pm 1, \pm 2\}$ are continuously differentiable and the series for $\hat{h}_{. \cdot}^{(j)}$ converges uniformly. The proof works in an absolute analogous way.

Finally we prove

Lemma 3.6. The terms $\widehat{p}_{l, j+1}$ can be bound by

$$
\begin{aligned}
\left\|\widehat{p}_{l, j+1}\right\|_{H_{s}^{0}} \leq C( & \left\|\widehat{R}_{-2, j+1}\right\|_{H_{s}^{0}}+\ldots+\left\|\widehat{R}_{2, j+1}\right\|_{H_{s}^{0}} \\
& \left.+\varepsilon^{1 / 2}\left(\left\|\widehat{R}_{-2, j+1}\right\|_{H_{s}^{0}}+\ldots+\left\|\widehat{R}_{2, j+1}\right\|_{H_{s}^{0}}\right)^{2}+1\right)
\end{aligned}
$$

and

$$
\left\|\widehat{p}_{l, j+1}-\widehat{p}_{l, j}\right\|_{H_{s}^{0}} \leq C q^{\frac{j}{2}}
$$

with $q \in(0,1)$ from Lemma 3.3 and with $C$ a constant independent of $j$ and $0<$ $\varepsilon \ll 1$.

Proof. Using (43) and Lemma 3.1 we find

$$
\begin{aligned}
\left\|\widehat{p}_{1, j+1}-\widehat{p}_{1, j}\right\|_{H_{s}^{0}} \leq & \sum_{\lambda \in\{2,-2\}}\left\|\partial_{T} \widehat{g}_{1, \lambda}^{(j)}\right\|_{X^{s, \varepsilon}}\left\|\widehat{R}_{\lambda, j}\right\|_{H_{s}^{0}} \\
& +\sum_{\lambda \in\{2,-2\}}\left\|\widehat{g}_{1, \lambda}^{(j)}\right\|_{X^{s, \varepsilon}}\left\|\widehat{p}_{\lambda, j}\right\|_{H_{s}^{0}}+\left\|\widehat{r}_{1, j}\right\|_{H_{s}^{0}} .
\end{aligned}
$$


[DS06, Lemma 3.1] immediately implies

$$
\left\|\widehat{r}_{1, j}\right\|_{H_{s}^{0}} \leq C \sum_{l \in\{2,-2\}}\left\|\widehat{g}_{1 l}^{(j)}\right\|_{X^{s, \varepsilon}}\left\|\widehat{R}_{l, j}\right\|_{H_{s}^{0}},
$$

with $C$ independent of $j$. From Lemma 3.3 c) and Remark 3.5 it follows

$$
\left\|\widehat{p}_{, j+1}-\widehat{p}_{, j}\right\|_{H_{s}^{0}} \leq C q^{\frac{j+1}{2}}\left\|\widehat{R}_{, j}\right\|_{H_{s}^{0}}+C q^{\frac{j+1}{2}}\left\|\widehat{p}_{, j}\right\|_{H_{s}^{0}}
$$

where $\widehat{p}_{, j}=\left(\widehat{p}_{-2, j}, \widehat{p}_{-1, j}, \widehat{p}_{1, j}, \widehat{p}_{2, j}\right)$. This inequality implies that $\left(\widehat{p}_{, j}\right)_{j \in \mathbb{N}}$ converges like a geometric series and that the $\widehat{p}_{, j}$ can be estimated in terms of $\widehat{p}_{, 1}$.

\section{The transformed equations and the final energy estimates}

Due to Lemma 3.3 and Lemma 3.6 we have for $l \in\{ \pm 1, \pm 2\}$ that $\left\|\widehat{f}_{l, \text { non }}^{(j)}(\cdot, \varepsilon t)\right\|_{X^{s, \varepsilon}} \rightarrow$ 0 for $j \rightarrow \infty$, the sequences $\left(\widehat{f}_{l, r e s}^{j)}(\cdot, \cdot, \varepsilon t)\right)_{j \in \mathbb{N}}$ are Cauchy sequences in $X^{s, \varepsilon}$ and that the sequences $\left(\widehat{p}_{l, j}(\cdot, \varepsilon t)\right)_{j \in \mathbb{N}}$ and $\left(\widehat{R}_{l, j}(\cdot, \varepsilon t)\right)_{j \in \mathbb{N}}$ are Cauchy sequences in $H_{s}^{0}$. From the completeness of $X^{s, \varepsilon}$ and $H_{s}^{0}$ it follows that their limits exist in $X^{s, \varepsilon}$ and $H_{s}^{0}$, respectively. Hence, after the infinitely many transformations we can eliminate the non-resonant terms in (21)-24) at leading order in $\varepsilon$. We arrive at

$$
\begin{aligned}
\partial_{t} \widehat{\mathcal{R}}_{1}(k, t)= & i \omega_{1}(k) \widehat{\mathcal{R}}_{1}(k, t) \\
& +i \omega_{1}(k) \int \sum_{\mu \in\{1,-1\}} \widehat{f}_{1, r e s}(k, k-m, \varepsilon t) \widehat{\mathcal{R}}_{\mu}(m, t) d m+\varepsilon \widehat{P}_{1}(k, t), \\
\partial_{t} \widehat{\mathcal{R}}_{-1}(k, t)= & -i \omega_{1}(k) \widehat{\mathcal{R}}_{-1}(k, t) \\
& -i \omega_{1}(k) \int \sum_{\mu \in\{1,-1\}} \widehat{f}_{1, r e s}(k, k-m, \varepsilon t) \widehat{\mathcal{R}}_{\mu}(m, t) d m+\varepsilon \widehat{P}_{-1}(k, t), \\
\partial_{t} \widehat{\mathcal{R}}_{2}(k, t)= & i \omega_{2}(k) \widehat{\mathcal{R}}_{2}(k, t) \\
& +i \omega_{2}^{-1}(k) \int \sum_{\mu \in\{2,-2\}} \widehat{f}_{2, r e s}(k, k-m, \varepsilon t) \widehat{\mathcal{R}}_{\mu}(m, t) d m+\varepsilon \widehat{P}_{2}(k, t), \\
\partial_{t} \widehat{\mathcal{R}}_{-2}(k, t)= & -i \omega_{2}(k) \widehat{\mathcal{R}}_{-2}(k, t) \\
& -i \omega_{2}^{-1}(k) \int \sum_{\mu \in\{2,-2\}} \widehat{f}_{2, r e s}(k, k-m, \varepsilon t) \widehat{\mathcal{R}}_{\mu}(m, t) d m+\varepsilon \widehat{P}_{-2}(k, t),
\end{aligned}
$$


where $\widehat{\mathcal{R}}_{l}, \widehat{f}_{l, \text { res }}, \widehat{P}_{l}$ are the limits of $\widehat{R}_{l, j}, \widehat{f}_{l, r e s}^{(j)}, \widehat{p}_{l, j}$ for $j \rightarrow \infty$. The higher order terms can be estimated as

$$
\begin{aligned}
\left\|\widehat{P}_{l}\right\|_{H_{s}^{0}} \leq C( & \left\|\widehat{\mathcal{R}}_{-2}\right\|_{H_{s}^{0}}+\ldots+\left\|\widehat{\mathcal{R}}_{2}\right\|_{H_{s}^{0}} \\
& \left.+\varepsilon^{1 / 2}\left(\left\|\widehat{\mathcal{R}}_{-2}\right\|_{H_{s}^{0}}+\ldots+\left\|\widehat{\mathcal{R}}_{2}\right\|_{H_{s}^{0}}\right)^{2}+1\right) .
\end{aligned}
$$

Undoing the diagonalization 20 yields

$$
\begin{aligned}
& \partial_{t} \widehat{\mathcal{R}}_{u}(k, t)=i \omega_{1}(k) \widehat{\mathcal{W}}_{u}(k, t)+\varepsilon \widehat{P}_{u_{1}}(k, t), \\
& \partial_{t} \widehat{\mathcal{W}}_{u}(k, t)=i \omega_{1}(k) \widehat{\mathcal{R}}_{u}(k, t)+i \omega_{1}(k) \int \widehat{f}_{u}(k, k-m, \varepsilon t) \widehat{\mathcal{R}}_{u}(m, t) d m+\varepsilon \widehat{P}_{u_{2}}(k, t), \\
& \partial_{t} \widehat{\mathcal{R}}_{v}(k, t)=i \omega_{2}(k) \widehat{\mathcal{W}}_{v}(k, t)+\varepsilon \widehat{P}_{v_{1}}(k, t), \\
& \partial_{t} \widehat{\mathcal{W}}_{v}(k, t)=i \omega_{2}(k) \widehat{\mathcal{R}}_{v}(k, t)+i \omega_{2}^{-1}(k) \int \widehat{f}_{v}(k, k-m, \varepsilon t) \widehat{\mathcal{R}}_{v}(m, t) d m+\varepsilon \widehat{P}_{v_{2}}(k, t),
\end{aligned}
$$

with the abbreviation

$$
\widehat{f}_{u}(k, k-m, \varepsilon t)=2 \widehat{f}_{1, r e s}(k, k-m, \varepsilon t), \quad \widehat{f}_{v}(k, k-m, \varepsilon t)=2 \widehat{f}_{2, r e s}(k, k-m, \varepsilon t)
$$

and

$$
\begin{aligned}
\left\|\widehat{P}_{w}\right\|_{H_{s}^{0}} \leq C( & \left\|\widehat{\mathcal{R}}_{u}\right\|_{H_{s}^{0}}+\ldots+\left\|\widehat{\mathcal{W}}_{v}\right\|_{H_{s}^{0}} \\
& \left.+\varepsilon^{1 / 2}\left(\left\|\widehat{\mathcal{R}}_{u}\right\|_{H_{s}^{0}}+\ldots+\left\|\widehat{\mathcal{W}}_{v}\right\|_{H_{s}^{0}}\right)^{2}+1\right)
\end{aligned}
$$

for $w \in\left\{u_{1}, u_{2}, v_{1}, v_{2}\right\}$ and $C$ only depending on $\omega_{1}, \omega_{2}$ and $\widehat{\Psi}$.

The energy estimates for (1) are based on integration by parts. The following lemma allows to transfer this approach to (56).

Lemma 4.1. For $w \in\{u, v\}$ the functions $\widehat{f}_{w}(k, k-m, \varepsilon t)$ satisfy:

$$
\begin{aligned}
& \text { (i) } \widehat{f}_{w}(k, k-m, \varepsilon t)=\overline{\widehat{f}_{w}(k, m-k, \varepsilon t)}, \\
& \text { (ii) } \sup _{k \in \mathbb{R}}\left\|\widehat{f}_{w}(k, \cdot, \varepsilon t)-\widehat{f}_{w}(k-\cdot, \cdot, \varepsilon t)\right\|_{L^{1}} \leq C \varepsilon .
\end{aligned}
$$

Proof. The starting point of the iteration process was

$$
\widehat{f}_{\lambda, r e s}^{(1)}(k, k-m, \varepsilon t)=\widehat{\Psi}(k-m, \varepsilon t),
$$


where $\widehat{\Psi}(\cdot, \varepsilon t)$ is the Fourier transform of a real-valued function, such that $\widehat{\Psi}(k, \cdot)=$ $\widehat{\Psi}(-k, \cdot)$. Therefore, we obtain

$$
\begin{aligned}
& \widehat{f}_{\lambda, r e s}^{(j)}(k, k-m, \varepsilon t)=\overline{\widehat{f}_{\lambda, r e s}^{(j)}(k, m-k, \varepsilon t)}, \\
& \widehat{f}_{\lambda, \text { non }}^{(j)}(k, k-m, \varepsilon t)=\overline{\widehat{f}_{\lambda, \text { non }}^{(j)}(k, m-k, \varepsilon t)}
\end{aligned}
$$

for $j=1$. Using (40) we find that $\widehat{g}_{\lambda \mu}^{(1)}(k, k-m, \varepsilon t)=\overline{\widehat{g}_{\lambda \mu}^{(1)}(k, m-k, \varepsilon t)}$ and hence

$$
\widehat{h}_{\lambda \mu}^{(1)}(k, k-m, \varepsilon t)=\overline{\widehat{h}_{\lambda \mu}^{(1)}(k, m-k, \varepsilon t)} .
$$

With the help of (41) and a simple induction, it follows that (57) holds for all $j \in \mathbb{N}$. Hence, the assertion in (i) is valid.

Property (ii) holds due the concentration of $\widehat{\psi}_{n}$ at the wave number $k=0$. In detail, we have

$$
\begin{aligned}
& \sup _{k \in \mathbb{R}}\left\|\widehat{f}_{w}(k, \cdot, \varepsilon t)-\widehat{f}_{w}(k-\cdot, \cdot, \varepsilon t)\right\|_{L^{1}} \\
\leq & \int \sup _{\xi \in \mathbb{R}}\left|\partial_{1} \widehat{f}_{w}(\xi, l)\right|\left(1+(l / \varepsilon)^{2}\right)^{s / 2} \frac{l}{\left(1+(l / \varepsilon)^{2}\right)^{s / 2}} d l \\
\leq & C \varepsilon\left\|\partial_{1} \widehat{f}_{w}\right\|_{X^{s, \varepsilon}} .
\end{aligned}
$$

due to Lemma 3.3 and Remark 3.5. This implies (ii).

In order to establish Theorem 1.1 we will prove an $\mathcal{O}(1)$-bound for an energy $\mathcal{E}_{s}$ which will be constructed in the following. It will be close to $E_{s}=E_{s, u}+E_{s, v}$ which is defined by

$$
\begin{array}{r}
E_{s, u}(t)=\sum_{j=0}^{s} \int|k|^{2 j}\left(\left|\widehat{\mathcal{R}}_{u}\right|^{2}(k, t)+\left|\widehat{\mathcal{W}}_{u}\right|^{2}(k, t)\right) d k, \\
E_{s, v}(t)=\sum_{j=0}^{s-1} \int|k|^{2 j} \omega_{2}^{2}(k)\left(\left|\widehat{\mathcal{R}}_{v}\right|^{2}(k, t)+\left|\widehat{\mathcal{W}}_{v}\right|^{2}(k, t)\right) d k .
\end{array}
$$

Computing $\frac{d}{d t} E_{s, u}(t)$ and realizing that the autonomous linear terms from 56 
cancel gives

$$
\begin{aligned}
\frac{d}{d t} E_{s, u}(t)= & \sum_{j=0}^{s} \iint|k|^{2 j} i \omega_{1}(k) \widehat{\widehat{\mathcal{W}}}_{u}(k, t) \widehat{f}_{u}(k, k-m, \varepsilon t) \widehat{\mathcal{R}}_{u}(m, t) d m d k \\
& -\sum_{j=0}^{s} \iint|k|^{2 j} i \omega_{1}(k) \widehat{\mathcal{W}}_{u}(k, t) \overline{\widehat{f}_{u}(k, k-m, \varepsilon t) \widehat{\mathcal{R}}_{u}(m, t)} d m d k+\varepsilon \widehat{Q}_{1}(t) \\
= & -\sum_{j=0}^{s} \iint|k|^{2 j} \partial_{t} \widehat{\mathcal{\mathcal { R }}}_{u}(k, t) \widehat{f}_{u}(k, k-m, \varepsilon t) \widehat{\mathcal{R}}_{u}(m, t) d m d k \\
& -\sum_{j=0}^{s} \iint|k|^{2 j} \partial_{t} \widehat{\mathcal{R}}_{u}(k, t) \overline{\widehat{f}_{u}(k, k-m, \varepsilon t) \widehat{\mathcal{R}}_{u}(m, t)} d m d k+\varepsilon \widehat{Q}_{2}(t)
\end{aligned}
$$

where we used the first line of $(56)$. Moreover, we have

$$
\left|\widehat{Q}_{l}\right| \leq C\left(E_{s}+\varepsilon^{1 / 2}\left(E_{s}\right)^{3 / 2}+1\right)
$$

for $l=1,2$. Interchanging the role of $k$ and $m$ in the second term yields

$$
\begin{aligned}
\frac{d}{d t} E_{s, u}(t)= & -\sum_{j=0}^{s} \iint|k|^{2 j} \partial_{t} \widehat{\mathcal{R}}_{u}(k, t) \widehat{f}_{u}(k, k-m, \varepsilon t) \widehat{\mathcal{R}}_{u}(m, t) d m d k \\
& -\sum_{j=0}^{s} \iint|m|^{2 j} \partial_{t} \widehat{\mathcal{R}}_{u}(m, t) \overline{\widehat{f}_{u}(m, m-k, \varepsilon t) \widehat{\mathcal{R}}_{u}(k, t)} d m d k+\varepsilon \widehat{Q}_{2}(t) .
\end{aligned}
$$

Using that $k^{j}=m^{j}+\mathcal{O}(k-m)$ and Lemma 4.1 gives next

$$
\begin{aligned}
\frac{d}{d t} E_{s, u}(t)= & -\sum_{j=0}^{s} \iint|k|^{j} \partial_{t} \widehat{\mathcal{R}}_{u}(k, t) \widehat{f}_{u}(k, k-m, \varepsilon t)|m|^{j} \widehat{\mathcal{R}}_{u}(m, t) d m d k \\
& -\sum_{j=0}^{s} \iint|m|^{j} \partial_{t} \widehat{\mathcal{R}}_{u}(m, t) \widehat{f}_{u}(m, k-m, \varepsilon t)|k|^{j} \widehat{\mathcal{R}}_{u}(k, t) d m d k+\varepsilon \widehat{Q}_{3}(t) \\
= & -\sum_{j=0}^{s} \iint|k|^{j} \partial_{t} \widehat{\mathcal{\mathcal { R }}}_{u}(k, t) \widehat{f}_{u}(k, k-m, \varepsilon t)|m|^{j} \widehat{\mathcal{R}}_{u}(m, t) d m d k \\
& -\sum_{j=0}^{s} \iint|m|^{j} \partial_{t} \widehat{\mathcal{R}}_{u}(m, t) \widehat{f}_{u}(k, k-m, \varepsilon t)|k|^{j} \overline{\widehat{\mathcal{R}}_{u}(k, t)} d m d k \\
& +\varepsilon Q_{4}(t)+\varepsilon \widehat{Q}_{3}(t),
\end{aligned}
$$


with

$\varepsilon Q_{4}(t)=-\sum_{j=0}^{s} \iint|m|^{j} \partial_{t} \widehat{\mathcal{R}}_{u}(m, t)\left(\widehat{f}_{u}(k, k-m, \varepsilon t)-\widehat{f}_{u}(m, k-m, \varepsilon t)\right)|k|^{j} \widehat{\mathcal{R}}_{u}(k, t) d m d k$

Hölder's inequality, Young's inequality, Lemma 4.1 and (56) allows us to prove for $Q_{3}$ and $Q_{4}$ the same estimates as for $Q_{1}$ and $Q_{2}$. Therefore we obtain

$$
\begin{aligned}
\frac{d}{d t} E_{s, u}(t) & =-\sum_{j=0}^{s} \iint \partial_{t}\left(|k|^{j} \widehat{\mathcal{\mathcal { R }}}_{u}(k, t)|m|^{j} \widehat{\mathcal{R}}_{u}(m, t)\right) \widehat{f}_{u}(k, k-m, \varepsilon t) d m d k+\varepsilon \widehat{Q}_{5}(t) \\
& =-\sum_{j=0}^{s} \partial_{t}\left(\iint|k|^{j} \widehat{\mathcal{R}}_{u}(k, t)|m|^{j} \widehat{\mathcal{R}}_{u}(m, t) \widehat{f}_{u}(k, k-m, \varepsilon t) d m d k\right)+\varepsilon \widehat{Q}_{6}(t)
\end{aligned}
$$

with

$$
\left|\widehat{Q}_{l}\right| \leq C\left(E_{s}+\varepsilon^{1 / 2}\left(E_{s}\right)^{3 / 2}+1\right)
$$

for $l=5,6$. Doing the same calculations for $E_{s, v}$ finally yields

$$
\partial_{t} \mathcal{E}_{s} \leq C \varepsilon\left(\mathcal{E}_{s}+\varepsilon^{1 / 2}\left(\mathcal{E}_{s}\right)^{3 / 2}+1\right)
$$

where

$$
\begin{aligned}
\mathcal{E}_{s}(t)= & E_{s}(t)+\sum_{j=0}^{s} \iint|k|^{j} \widehat{\mathcal{R}}_{u}(k, t)|m|^{j} \widehat{\mathcal{R}}_{u}(m, t) \widehat{f}_{u}(k, k-m, \varepsilon t) d m d k \\
& +\sum_{j=0}^{s} \iint|k|^{j} \widehat{\mathcal{R}}_{v}(k, t)|m|^{j} \widehat{\mathcal{R}}_{v}(m, t) \widehat{f}_{v}(k, k-m, \varepsilon t) d m d k .
\end{aligned}
$$

Now, a simple application of Gronwall's inequality yields an $\mathcal{O}(1)$-bound for $\mathcal{E}_{s}$ for all $t \in\left[0, T_{0} / \varepsilon\right]$ for $\varepsilon>0$ sufficiently small. Since $\left\|R_{u}\right\|_{H^{s}}+\left\|R_{v}\right\|_{H^{s}}+\left\|W_{u}\right\|_{H^{s}}+\left\|W_{v}\right\|_{H^{s}} \leq$ $\sqrt{\mathcal{E}_{s}}$ for $\Psi$ sufficiently small we obtain

$$
\sup _{t \in\left[0, T_{0} / \varepsilon\right]}\|(u, v)(\cdot, t)-(U, V)(\varepsilon \cdot, \varepsilon t)\|_{H^{s}}=\varepsilon^{3 / 2} \sup _{t \in\left[0, T_{0} / \varepsilon\right]}\|R(\cdot, t)\|_{H^{s}} \leq C \varepsilon^{3 / 2} .
$$

With the help of Sobolev's embedding theorem we finally get the bound stated in Theorem 1.1. 


\section{Discussion}

The question occurs whether to a given initial condition the associated solution $(u, v)$ of the KGB system can be approximated by the Whitham approximation.

Theorem 5.1. Consider the KGB system (2)-(3) with the family of initial conditions

$$
\left(u, \partial_{t} u, v, \partial_{t} v\right)(x, 0)=\left(\Phi_{1}, \varepsilon \Phi_{2}, H\left(\Phi_{1}\right), \varepsilon H^{\prime}\left(\Phi_{1}\right) \Phi_{2}\right)(\varepsilon x)
$$

parametrized by $\varepsilon>0$. Then for every $T_{0}>0$ there exist $C>0$ and $\varepsilon_{0}>0$ such that for $\Phi_{j}$ satisfying $\left\|\Phi_{1}\right\|_{H^{5}}+\left\|\partial_{X}^{-1} \Phi_{2}\right\|_{H^{5}} \leq C$ and every $\varepsilon \in\left(0, \varepsilon_{0}\right)$ there are solutions $U \in C\left(\left[0, T_{0}\right], H^{5}\right)$ of the Whitham equation (6) with initial conditions $U(X, 0)=\Phi_{1}(X)$ and $\partial_{T} U(X, 0)=\Phi_{2}(X)$ such that

$$
\sup _{t \in\left[0, T_{0} / \varepsilon\right]}\|(u, v)(\cdot, t)-(U, H(U))(\varepsilon \cdot, \varepsilon t)\|_{H^{1}} \leq C_{2} \varepsilon^{3 / 2} .
$$

Remark 5.2. The previous assumptions on $v$ can be weakened slightly to

$$
\left\|\left(v, \varepsilon^{-1} \partial_{t} v\right)(\cdot, 0)-\left(H\left(\Phi_{1}\right), H^{\prime}\left(\Phi_{1}\right) \Phi_{2}\right)(\varepsilon \cdot)\right\|_{H^{5} \times H^{4}} \leq C \varepsilon^{3 / 2} .
$$

The proof of Theorem 5.1 and of Remark 5.2 is a direct consequence of the estimates shown in Section 4. The local existence and uniqueness of solutions for the Whitham equation (6) resp. for (7)-(8) follows from [Kat75]. It is not expected that the set of initial conditions for the KGB system for which the associated solutions can be described by the Whitham approximation is much larger.

\section{References}

[BSTU06] K. Busch, G. Schneider, L. Tkeshelashvili, H. Uecker. Justification of the nonlinear Schrödinger equation in spatially periodic media. Z. Angew. Math. Phys., 57(6):905-939, 2006.

[CCPS12] M. Chirilus-Bruckner, C. Chong, O. Prill, G. Schneider. Rigorous description of macroscopic wave packets in infinite periodic chains of coupled oscillators by modulation equations. $D C D S-S 5(5): 879-901,2012$.

[CS11] C. Chong, G. Schneider. The validity of the KdV approximation in case of resonances arising from periodic media. J. Math. Anal. Appl., 383(2):330-336, 2011. 
[CE90] P. Collet, J.-P. Eckmann. The time dependent amplitude equation for the Swift-Hohenberg problem. Comm. Math. Phys., 132(1):139-153, 1990.

[Cra85] W. Craig. An existence theory for water waves and the Boussinesq and Korteweg-de Vries scaling limits. Comm. Partial Differential Equations, 10(8):787-1003, 1985.

[DSSS09] A. Doelman, B. Sandstede, A. Scheel, G. Schneider. The dynamics of modulated wave trains. Mem. Amer. Math. Soc., 199(934):viii+105, 2009.

[DHM06] W. Dreyer, M. Herrmann, A. Mielke. Micro-macro transition in the atomic chain via Whitham's modulation equation. Nonlinearity 19(2):471-500, 2006.

[D12] W.-P. Düll. Validity of the Korteweg-de Vries Approximation for the TwoDimensional Water Wave Problem in the Arc Length Formulation. Comm. Pure Appl. Math. 65(3):381-429, 2012.

[DS06] W.-P. Düll, G. Schneider. Justification of the Nonlinear Schrödinger equation for a resonant Boussinesq model. Indiana Univ. Math. J. 55(6):1813-1834, 2006.

[DS09] W.-P. Düll, G. Schneider. Validity of Whitham's equations for the modulation of periodic traveling waves in the NLS equation. J. Nonlinear Sci., 19(5):453-466, 2009.

[DSW16] W.-P. Düll, G. Schneider, C. E. Wayne. Justification of the Nonlinear Schrödinger equation for the evolution of gravity driven 2D surface water waves in a canal of finite depth. Arch. Rat. Mech. Anal., 220(2):543-602, 2016.

[GMWZ14] J. Gaison, S. Moskow, J. D. Wright, Q. Zhang. Approximation of polyatomic FPU lattices by KdV equations. Multiscale Model. Simul. 12(3):953-995, 2014.

[GS01] T. Gallay, G. Schneider. KP description of unidirectional long waves. The model case. Proc. R. Soc. Edinb., Sect. A, Math. 131(4):885-898, 2001.

[JNRZ14] M. A. Johnson, P. Noble, L. M. Rodrigues, K. Zumbrun. Behavior of periodic solutions of viscous conservation laws under localized and nonlocalized perturbations. Invent. Math., 197(1):115-213, 2014.

[Kal87] L. A. Kalyakin. Asymptotic decay of a one-dimensional wave packet in a nonlinear dispersive medium. Math. USSR, Sb. 60(2):457-483, 1988; translation from Mat. Sb., Nov. Ser. 132(174), No.4, 470-495, 1987. 
[Kat75] T. Kato. Quasi-linear equations of evolution, with applications to partial differential equations in: Spectral Theory and Differential Equations. Lecture Notes in Mathematics, 448:25-70, 1975.

[MS04a] I. Melbourne, G. Schneider. Phase dynamics in the complex GinzburgLandau equation. J. Differential Equations, 199(1):22-46, 2004.

[MS04b] I. Melbourne, G. Schneider. Phase dynamics in the real Ginzburg-Landau equation. Math. Nachr., 263/264:171-180, 2004.

[M06] A. Mielke. Macroscopic behavior of microscopic oscillations in harmonic lattices via Wigner-Husumi transforms. Arch. Rat. Mech. Anal., 181:401-448, 2006.

[Sch94a] G. Schneider. A new estimate for the Ginzburg-Landau approximation on the real axis. J. Nonlinear Sci., 4(1):23-34, 1994.

[Sch94b] G. Schneider. Error estimates for the Ginzburg-Landau approximation. Z. Angew. Math. Phys., 45(3):433-457, 1994.

[Sch95] G. Schneider. Validity and limitation of the Newell-Whitehead equation. Math. Nachr., 176:249-263, 1995.

[Sch98] G. Schneider. Justification of modulation equations for hyperbolic systems via normal forms. NoDEA Nonlinear Differential Equations Appl., 5(1):69-82, 1998.

[Sch05] G. Schneider. Justification and failure of the nonlinear Schrödinger equation in case of non-trivial quadratic resonances. J. Differential Equations, 216(2):354$386,2005$.

[SSZ15] G. Schneider, D. A. Sunny, and D. Zimmermann. The NLS approximation makes wrong predictions for the water wave problem in case of small surface tension and spatially periodic boundary conditions. Journal of Dynamics and Differential Equations 27(3):1077-1099, 2015.

[SU16] G. Schneider, H. Uecker. Nonlinear PDEs - A Dynamical Systems Approach via modulation equations-. 542 pages, submitted 2016.

[SW00a] G. Schneider, C. E. Wayne. The long-wave limit for the water wave problem. I. The case of zero surface tension. Comm. Pure Appl. Math., 53(12):1475-1535, 2000 . 
[SW00b] G. Schneider, C. E. Wayne. Counter-propagating waves on fluid surfaces and the continuum limit of the Fermi-Pasta-Ulam model. In International Conference on Differential Equations, Vol. 1, 2 (Berlin, 1999), pages 390-404. World Sci. Publishing, River Edge, NJ, 2000.

[SW02] G. Schneider, C. E. Wayne. The rigorous approximation of long-wavelength capillary-gravity waves. Arch. Ration. Mech. Anal., 162(3):247-285, 2002.

[TW12] N. Totz, Sijue Wu. A rigorous justification of the modulation approximation to the 2D full water wave problem. Commun. Math. Phys., 310(3):817-883, 2012.

[vH91] A. van Harten. On the validity of the Ginzburg-Landau equation. J. Nonlinear Sci., 1(4):397-422, 1991.

[Whi65a] G. B. Whitham. A general approach to linear and non-linear dispersive waves using a Lagrangian. J. Fluid Mech., 22:273-283, 1965.

[Whi65b] G. B. Whitham. Non-linear dispersive waves. Proc. Roy. Soc. Ser. A, 283:238-261, 1965. 